\title{
De l'importance du suivi pour maîtriser le dimensionnement des ouvrages géotechniques ${ }^{1}$
}

\author{
P. SCHMITT \\ Solétanche-Bachy \\ 133, bd National \\ BP 90250 \\ 92504 Rueil-Malmaison
}

Cet article a pour but de mettre en évidence, à partir d'un nombre significatif d'exemples plus ou moins récents, la nécessité de suivre de façon systématique le comportement réel des ouvrages géotechniques; on entend ici par suivi non pas l'observation passive, mais la mesure et l'exploitation des mesures, instantanée ou différée, selon une procédure à définir au cas par cas en fonction de l'incertitude et des risques que le concepteur de l'ouvrage se doit d'avoir analysés au préalable. On s'attache dans un premier temps à montrer les limites du calcul géotechnique, face à la complexité et aux exigences croissantes imposées par l'environnement visà-vis de la conception des ouvrages géotechniques. On montre dans un deuxième temps comment le suivi d'ouvrages géotechniques pratiqué lors des dernières décennies (principalement des écrans de soutènement) a pu permettre de valider les approches de calcul classiques reposant sur la notion de coefficient de réaction du sol, de préciser les ordres de grandeur des paramètres associés, de mieux connaître les vraies limites de ces approches simplifiées, et d'apporter un éclairage sur l'utilisation de méthodes numériques de portée plus générale. On donne enfin quelques exemples montrant comment un suivi interactif permet de mieux maîtriser la sécurité et l'optimisation des ouvrages, et comment la teneur et la gestion de ce suivi peuvent être modulées en fonction des risques de dépassement d'états limites, que les analyses préalablement effectuées par le concepteur de l'ouvrage ont normalement permis de détecter.

Mots-clés : méthode observationnelle, dimensionnement interactif, écrans de soutènement, rétro-analyses, coefficient de réaction, modèles de comportement, raideur du sol, états limites, analyse de risques.

\section{Monitoring geotechnical structures : a requirement for design}

This article intends to demonstrate, using a significant number of classical or recent examples, the need for a systematic monitoring of geotechnical structures ; monitoring is considered here as an active process, including continuous measurements and instantaneous or not back-analysis, depending on

preliminary evaluation of uncertainties and risks.

The first part shows geotechnical calculation limits, compared with continuously increasing complexity and constraints imposed by environmental requirements.

The second part shows how several decades of geotechnical monitoring, more especially in the field of retaining structures, made it possible to validate conventional calculation models such as those resting on subgrade reaction theory, to set relevant orders of magnitude for associated parameters, to better know actual limits of such simplified approaches, and also to provide useful information to calibrate more general numerical models.

(1) Texte de la conférence Coulomb prononcée lors de la séance technique du CFMS le 12 juin 2008. 
The third part finally gives examples showing how interactive monitoring makes it possible to better guarantee both safe and optimized designs, and how monitoring process itself may be adjusted in order not to exceed any of the potential limit states detected by preliminary analysis.

Key words: observational method, interactive design, retaining structures, back-analysis, subgrade reaction modulus, soil models, soil stiffness, limit states, risk analysis.

\section{Introduction}

La nécessité de confronter les théories à la réalité, en géotechnique comme dans tous les domaines de l'ingénierie, est une évidence dans l'esprit de tous, et ce principe a toujours fait partie de l'enseignement même de la géotechnique.

Force est pourtant de constater que la pratique intègre parfois difficilement cette évidence, dont la prise en compte pourrait être perçue comme une entrave au déroulement optimal des travaux : il est intellectuellement plus confortable, et en première approximation plus efficace, de fonctionner dans un univers virtuel constitué de certitudes inébranlables, ce qui explique qu'au-delà des bonnes intentions, la pratique soit parfois peu réceptive à ce qui risquerait de remettre en cause les fondements, réglementaires ou bibliographiques, sur lesquels repose la conception du projet.

Cet article vise pourtant à montrer que l'évolution des projets et l'expérience acquise au cours des 30 dernières années mettent en lumière non seulement l'importance, mais encore l'absolue nécessité de ce qu'il est convenu d'appeler le suivi géotechnique.

On présente ainsi successivement :

- quelques réflexions mettant en évidence les limites du calcul géotechnique ;

- quelques exemples démontrant l'intérêt du suivi géotechnique pour améliorer la prévision du comportement réel des ouvrages (principalement des écrans de soutènement) ;

- quelques exemples visant à montrer l'intérêt du suivi géotechnique pour améliorer la maîtrise de la sécurité des ouvrages, dans le cadre de ce qu'il est convenu d'appeler la méthode observationnelle, version plus contraignante du suivi géotechnique, plus justement décrite par le terme " dimensionnement interactif » (Allagnat, 2005; Schlosser et Schmitt, 2007).

\section{2}

\section{Les limites du calcul géotechnique}

On est souvent trop peu conscient de l'évolution considérable, en termes d'exigence vis-à-vis du calcul, générée par l'évolution continue des projets au cours des 30 dernières années.

Le calcul géotechnique des années 70 reposait essentiellement sur des schémas conventionnels issus de la théorie du calcul à la rupture (capacité portante des fondations, équilibre de poussée-butée des écrans de soutènement, etc.) : les dimensionnements étaient donc implicitement établis par référence à des états limites ultimes, dont on se maintenait éloignés en pratique par l'usage de coefficients de sécurité élevés.
Ces coefficients de sécurité étaient en partie explicites ( (aux de travail du sol » n'excédant pas le tiers de la charge limite par exemple), en partie implicites (hypothèses de calcul réputées sécuritaires), d'où résultait la conviction générale que le calcul géotechnique était largement « dans le sens de la sécurité », au point qu'il pouvait même sembler frustrant, voire coûteux, de faire reposer la conception des ouvrages sur des calculs aussi peu représentatifs.

Les coefficients de sécurité de l'époque étaient même réputés suffisamment élevés pour dispenser, en général, de vérifications approfondies en termes de déplacements.

Ainsi l'apparition dans les bureaux d'études, vers la fin des années 70 , des premiers programmes de calculs aux coefficients de réaction, permettant de mieux appréhender qu'auparavant la déformée des écrans de soutènement, a été avant tout perçue comme un moyen de mieux aborder le calcul des sollicitations : le déplacement était un résultat annexe intéressant, mais il n'intervenait pas explicitement dans la procédure de dimensionnement, tant était enracinée dans la pensée dominante de l'époque la conviction que ces déplacements calculés étaient toujours bien supérieurs aux déplacements réels.

Ceci étant, comme c'est souvent le cas, l'évolution des projets dans la période récente s'est avérée beaucoup plus rapide que celle des méthodes de calcul, sous la pression de plusieurs facteurs déterminants parmi lesquels on peut citer :

- la saturation progressive des sites disponibles pour les implantations industrielles ou immobilières, conduisant à travailler dans des terrains aux caractéristiques de plus en plus médiocres, et souvent dans un environnement urbain de plus en plus contraignant, en termes notamment d'exiguïté du site et de sensibilité des ouvrages existants ;

- les progrès technologiques permettant de réaliser des ouvrages enterrés de plus en plus profonds, subissant donc de la part du sol des sollicitations de plus en plus importantes ;

- le développement des entreprises de travaux spéciaux et de la concurrence associée, conduisant naturellement à renforcer leur compétitivité à travers non seulement des innovations techniques, mais encore à travers l'optimisation continue des dimensionnements, ce qui suppose inévitablement une approche plus rigoureuse de la sécurité.

Corrélativement, l'encadrement réglementaire s'est lui-même développé jusqu'à l'apparition récente des Eurocodes structuraux et de leurs annexes nationales.

Curieusement, une idée couramment répandue est que, dans le domaine de la géotechnique, l'évolution majeure serait la référence accrue aux états limites ultimes, avec l'introduction explicite de coefficients de sécurité partiels, qui n'existaient souvent auparavant, 
il est vrai, que sous une forme globale ou implicite, alors qu'en réalité l'évolution majeure de ces dernières années est bel et bien la référence aux états limites de service, qui se traduit en premier lieu par l'introduction explicite de nouveaux critères de dimensionnement exprimés en termes de déplacements admissibles.

Le calcul des ouvrages géotechniques repose donc bien dorénavant sur deux préoccupations distinctes, qui sont la sécurité et les déformations, que l'on n'accepte plus d'amalgamer comme auparavant, sous couvert d'un calcul approximatif dont l'imprécision serait compensée par l'introduction plus ou moins explicite de coefficients globaux.

Au contraire, la vérification explicite d'un état limite de service exprimé sous forme d'un critère de déplacement constitue une contrainte doublement draconienne:

- la vérification par le calcul ne comporte plus aucun coefficient de sécurité, qu'il soit explicite, comme c'est le cas pour la vérification d'un état limite ultime, ou implicite, dans la mesure où les valeurs caractéristiques des paramètres de calcul sont censées n'être que des valeurs moyennes (pour un volume de sol réputé homogène) représentant, de façon prudente mais aussi réaliste que possible, les propriétés de la fraction du massif de sol qui se trouve en interaction avec l'ouvrage ;

- contrairement au cas d'un état limite structurel, qui ne va généralement pas donner lieu à une vérification in situ (la mesure précise de l'ouverture réelle des fissures d'un ouvrage en béton armé fait rarement partie des critères de réception de cet ouvrage), il est bien évident que le maintien en service des constructions existantes, qu'il est indispensable d'assurer, dépend directement des déformations réelles, et non pas théoriques du sol, et que le respect du critère de déplacement faisant l'objet du calcul va donc de plus en plus souvent faire l'objet d'un contrôle in situ.

Il en résulte que l'on demande, dorénavant, à ce même calcul dont l'imprécision reconnue était auparavant réputée compensée par l'usage de coefficients de sécurité élevés, de fournir, avec un coefficient de sécurité égal à 1 , la valeur réelle exacte de ce qui est le plus difficile à appréhender en géotechnique, à savoir la déformation du sol !

Or qu'a-t-on fait en 30 ans pour accompagner cette exigence déraisonnablement accrue vis-à-vis du calcul?

Les reconnaissances géotechniques ne semblent pas avoir évolué dans le sens d'une densité plus importante de sondages et d'essais, pourtant indispensables pour mieux cerner les valeurs réelles des paramètres représentatifs du comportement du sol ; au contraire, il est regrettable d'avoir assisté durant cette période à une réduction drastique de l'importance des essais de laboratoire, pourtant seuls capables d'appréhender les lois de comportements complexes dont la maîtrise serait indispensable pour permettre la réalisation de calculs réellement représentatifs.

La seule évolution significative est celle, non pas des méthodes de calcul, qui n'ont pour ainsi dire pas évolué en 30 ans, mais de la puissance des ordinateurs et de la convivialité des logiciels, qui permettent de mettre dorénavant entre toutes les mains des outils très spécialisés, extrêmement complexes et sensibles à des variations mineures de paramètres que plus personne ne mesure!
Ceci explique la persistance, dans la pratique, de méthodes de dimensionnement empiriques, au sens noble du terme, c'est-à-dire basées sur l'expérience et l'observation; le problème de ces approches est qu'une méthode empirique n'est par définition valable que dans le domaine expérimental nécessairement limité qui a permis de l'établir, et que ce domaine n'est jamais explicitement précisé, alors qu'il est évident que la précision d'une approche empirique décroît très fortement, et même dangereusement, au fur et à mesure que l'on s'approche des frontières de son domaine de validité.

Le praticien a donc aujourd'hui à sa disposition, pour fournir les résultats des calculs de plus en plus précis sur lesquels on lui demande de s'engager, soit des méthodes de calcul numériques très performantes mais qui exigent de lui la connaissance de lois complexes et des paramètres associés, que personne n'est en mesure de lui communiquer, soit des méthodes empiriques dont il ignore le domaine de validité et dont il est par conséquent parfaitement incapable de connaître la précision réelle.

Il est important d'insister sur le fait que l'imprécision du calcul ne provient finalement qu'en partie de l'hétérogénéité du sol, laquelle pourrait en théorie être maîtrisée par des reconnaissances géotechniques plus complètes, réalisées suffisamment en amont des études, et complétées comme il se doit au fur et à mesure de l'avancement du projet, conformément à la norme française relative aux missions d'ingénierie géotechnique (AFNOR, 2006), ainsi que par une détermination plus rigoureuse des valeurs caractéristiques des paramètres de comportement; mais aucun texte ne traite aujourd'hui le problème, encore plus redoutable, de la précision des modèles de calcul eux-mêmes, et de l'incertitude inhérente à la méconnaissance générale de leurs domaines de validité réels.

Comment expliquer, dans ces conditions, que la conception et la réalisation d'ouvrages géotechniques de plus en plus complexes et audacieux ne soient pas devenues des aventures totalement hasardeuses?

La seule réponse possible réside dans la prise de conscience progressive, et dans la mise en œuvre, également progressive, envers et contre tout, par les principaux acteurs français et européens de la profession, du « suivi géotechnique », jusqu'à son aboutissement qu'est le « dimensionnement interactif », dorénavant érigé par le guide français établi dans le cadre de l'IREX (Allagnat, 2005), et par l'Eurocode 7 lui-même en méthode de dimensionnement à part entière.

Seule une telle approche pragmatique a pu permettre de pallier l'incertitude inhérente aux différents modèles de calcul, en permettant à la fois :

- de la réduire, par l'observation continue des ouvrages pratiquée dans le cadre du suivi géotechnique (cf. § 3) ; - de la gérer, en recourant chaque fois que nécessaire à la méthode du dimensionnement interactif (cf. § 4).

3

\section{Prévision du comportement réel des écrans de soutènement}

Le calcul des écrans de soutènement fait depuis 30 ans en France l'objet de polémiques entre les partisans des différentes approches, alors que le vrai 
problème n'est finalement pas tant celui de la validité d'une méthode que celui de son domaine d'utilisation.

Or chaque méthode est associée à un modèle de comportement de l'ouvrage et du sol, et c'est donc la connaissance de ces modèles, déduite de l'expérience et des observations, qui peut seule permettre de préciser les domaines de validité réels des méthodes de calcul associées.

Ainsi les modèles de calcul reposant sur la seule prise en compte de l'équilibre limite de poussée-butée des écrans de soutènement, que plus personne n'utilise aujourd'hui alors que ce sont les seuls capables de prédimensionner efficacement un ouvrage par référence aux états limites d'instabilité rotationnelle décrits par l'Eurocode 7, ont un domaine de validité probablement limité aux ouvrages isostatiques, bien qu'ils aient dans le passé permis de réaliser avec succès des ouvrages plus complexes.

Les modèles de type poutre sur appuis élastiques, dits ( aux coefficients de réaction », sont parfois réputés n'avoir qu'un domaine de validité quasi inexistant en raison de la fragilité de leur support théorique, alors que la pratique française des 30 dernières années a démontré au contraire, à la surprise générale, leur efficacité dans le domaine non seulement du dimensionnement, mais encore de la prévision du comportement des ouvrages courants.

La méthode des éléments finis, réputée universelle, est la plus prometteuse dans la mesure où son usage systématique permettrait en théorie d'éluder le problème de la connaissance des modèles de comportement particuliers et des limites d'utilisation des méthodes empiriques associées, qui reposent nécessairement sur la prise en compte de mécanismes simplifiés.

Cependant, cette généralisation butte toujours à l'heure actuelle sur deux problèmes majeurs qui sont la connaissance, insuffisante en pratique, des lois de comportement réellement représentatives du comportement des sols et des paramètres associés, d'une part, et d'autre part, sur l'absence de programme de calcul possédant des fonctionnalités suffisantes pour représenter correctement à la fois la construction, le fonctionnement et l'évolution dans le temps d'une structure enterrée complexe, le comportement réel du massif de sol entourant cette structure, et l'interaction entre les deux : ceci explique que la pratique consiste toujours à l'heure actuelle à enchaîner, dans la plupart des cas, des calculs modélisant séparément la structure, le massif de sol dans son ensemble, et l'interaction entre les deux, cette dernière faisant le plus souvent l'objet de calculs aux coefficients de réaction.

On s'attachera dans ce qui suit à mettre en évidence l'éclairage apporté par le suivi géotechnique sur les questions suivantes, toutes fondamentales pour la mise en œuvre pratique d'un modèle de calcul réellement représentatif du comportement réel de l'ouvrage :

- validation du modèle « poutre sur appuis élastiques », et calage des paramètres associés ;

- mise en évidence de quelques modèles de comportement élémentaires, non pris en compte par le modèle précédent, et dont l'observation permet de mieux situer les frontières de son domaine de validité ;

- calage d'une loi de comportement représentative du comportement réel du sol, dans l'optique de la mise en œuvre d'une modélisation numérique globale ;

- mise en évidence des limites générales des modélisations numériques elles-mêmes.
On ne saurait manquer de rendre ici hommage à l'initiateur de cette démarche qu'aura été Daniel Gouvenot, qui avait non seulement compris très rapidement l'importance pratique du suivi comme facteur de progrès pour le dimensionnement des ouvrages géotechniques, mais avait également su, avec toute la force de persuasion qui le caractérisait, faire progressivement passer cette notion fondamentale dans la culture de l'Entreprise (voir par exemple : Bustamante et Gouvenot, 1978).

\section{1}

\section{Mise en évidence du modèle " poutre sur appuis élastiques "}

Le succès de ce modèle depuis la fin des années 70 provient essentiellement de son intérêt pratique, dans la mesure où il permet, sans complication excessive et moyennant le choix d'un nombre limité de paramètres, une approche plus réaliste que les modèles traditionnels ne reposant que sur la théorie des équilibres limites, lesquels étaient (et sont toujours) bien adaptés à l'étude d'états limites géotechniques au sens de l'Eurocode 7, mais incapables de prendre en compte l'effet cumulé des chargements/déchargements successifs et les déformations réelles des ouvrages, donc peu adaptés à une évaluation réaliste des états limites structurels et des états limites de service.

Cependant ce modèle très pratique, et relativement classique puisque introduit par Winckler et Wertergaard dès les années 20 (Westergaard, 1926 ; Terzaghi, 1955), a longtemps été rejeté par la communauté scientifique, en raison du « scandale géotechnique ») sur lequel il reposait, consistant à assimiler le massif de sol à un ensemble de petits ressorts indépendants les uns des autres, ce qui suppose notamment une proportionnalité, contraire à la réalité, entre le déplacement et la contrainte en tout point de l'interface.

C'est donc exclusivement le suivi géotechnique pratiqué sur un nombre représentatif d'ouvrages réels qui aura permis de mettre en évidence la surprenante validité de ce modèle apparemment simpliste, ce qui explique qu'il soit devenu et demeure encore aujourd'hui un véritable standard pour le dimensionnement pratique des écrans de soutènement.

Il faut reconnaitre que les raisons de cette représentativité, incontestable puisque démontrée par de multiples observations, restent encore à éclaircir. On peut en effet concevoir qu'il soit licite d'approcher le comportement du sol, dans le domaine limité par le « bulbe de contraintes ) représentant la zone d'influence de l'ouvrage (Terzaghi, 1955), par un coefficient de réaction global représentant le rapport, dans ce domaine réputé homogène, de la contrainte moyenne à l'interface au déplacement moyen de cet interface, même si cette proportionnalité supposée n'est évidemment pas représentative du comportement local. Cependant, plusieurs questions restent alors en suspens :

- comment expliquer que le comportement d'un matériau notoirement non linéaire puisse être représenté par un paramètre unique reposant sur le schéma idéal d'un comportement à la fois élastique et linéaire ?

- comment expliquer que les différentes « zones d'influence ») du sol au voisinage d'un écran, sollicitées de façons très diverses (mise en butée élastique du massif amont sous l'effet d'ancrages précontraints, 
mise en butée du sol sous le niveau de fond de fouille pour mobiliser la réaction nécessaire à l'équilibre de l'écran...), puissent être représentées, en supposant le sol intrinsèquement homogène, par une valeur unique d'un coefficient de réaction pourtant inversement proportionnel à la hauteur de cette zone d'influence?

On verra ci-après comment le suivi géotechnique d'un nombre représentatif d'ouvrages réels a également permis d'apporter des éléments de réponse à ces questions fondamentales.

Rappelons au préalable la modélisation classique, proposée par Terzaghi (1955) et reprise par Ménard (Bourdon et Ménard, 1965), qui consiste à évaluer le coefficient de réaction du sol sollicité en butée élasti-

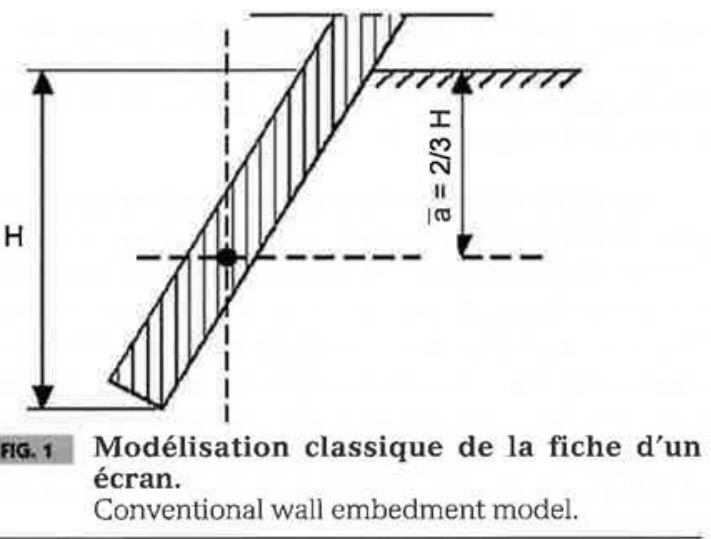

METRO DE LILLE

DEFORMATION DU RIDEAU DE PALPLANCHES

Tube inclinométrique $\mathrm{N}^{\circ} 4$

Etat initial: 20-3-81

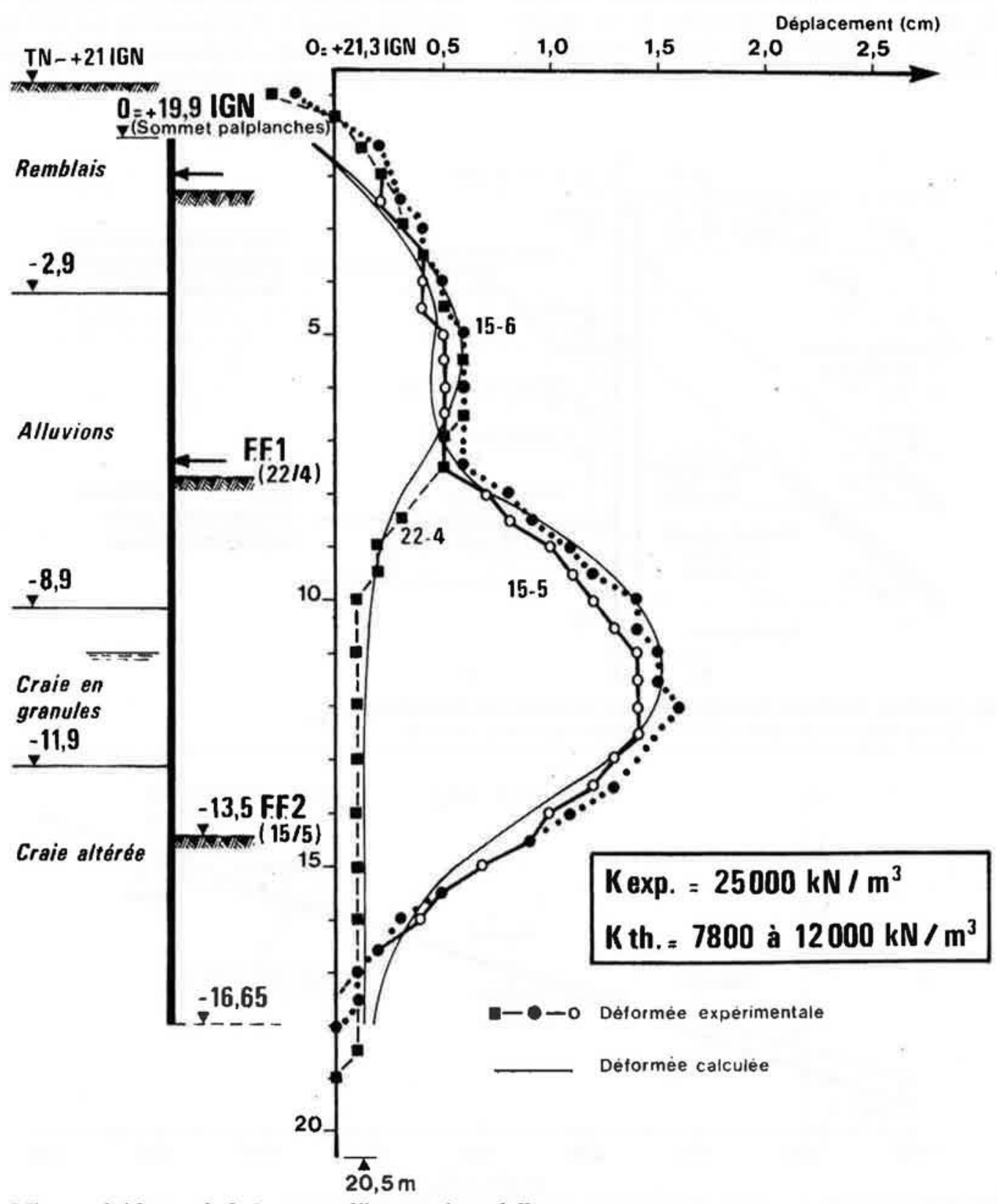

nG. 2 Mise en évidence de la hauteur d'interaction réelle.

Measurement of the actual interaction height. 
que par la fiche de l'écran en assimilant cette dernière à une fondation superficielle verticale (à l'anisotropie du sol près), dont l'axe coïnciderait avec le niveau du fond de fouille (Fig. 1).

La demi-largeur de cette semelle équivalente avait été initialement évaluée, par les auteurs précités, aux $2 / 3$ de la fiche totale, ce qui est légitime dans la mesure où les coefficients de sécurité usuels reviennent en pratique à rechercher un encastrement partiel par mobilisation d'une contre-butée vers la base de l'écran, ce qui justifie bien la prise en compte d'une partie seulement de la fiche totale dans l'évaluation de la zone d'influence du sol sollicité en butée sous le niveau de l'excavation.

Un premier intérêt du suivi géotechnique, pratiqué dès le début des années 80 , a été de mettre en évidence le fait que cette évaluation forfaitaire était inadaptée aux phases intermédiaires d'excavation de l'écran, pour lesquelles la hauteur située sous le niveau de l'excavation est bien supérieure et pour lesquelles la zone d'influence de l'écran se trouve en pratique bien souvent limitée à une profondeur dépendant plus de la rigidité relative de l'écran par rapport au sol, autrement dit de sa capacité à répartir les efforts, que de la hauteur totale de la fiche, dont la partie inférieure se trouve en pratique peu sollicitée (Fig. 2 ; Schmitt, 1984).

Cette approche plus réaliste de la zone d'influence de l'écran permettait en outre de comprendre, à condition d'admettre l'hypothèse de Terzaghi et Ménard consistant à assimiler la fiche de l'écran à une demisemelle sollicitée par l'effort tranchant transmis par la partie supérieure, pourquoi les différentes zones de mobilisation du sol de part et d'autre de l'écran pouvaient en pratique être représentées par une valeur unique du coefficient de réaction, inversement proportionnelle à une ( hauteur d'influence » ne dépendant elle-même dans le cas général que de la rigidité relative de l'écran par rapport au sol, constante pour une inertie d'écran et un module d'élasticité du sol donnés.

Restait à valider l'hypothèse hardie consistant à assimiler la fiche à une demi-semelle verticale sollicitée ponctuellement, ce qu'a également permis l'analyse du suivi géotechnique des ouvrages.

En effet, l'hypothèse retenue pour l'exploitation des mesures de déformation a consisté à définir forfaitairement la hauteur d'influence de l'écran comme la hauteur sur laquelle le déplacement était supérieur ou égal à $20 \%$ de sa valeur maximale (Fig. 3 ; Schmitt, 1984).

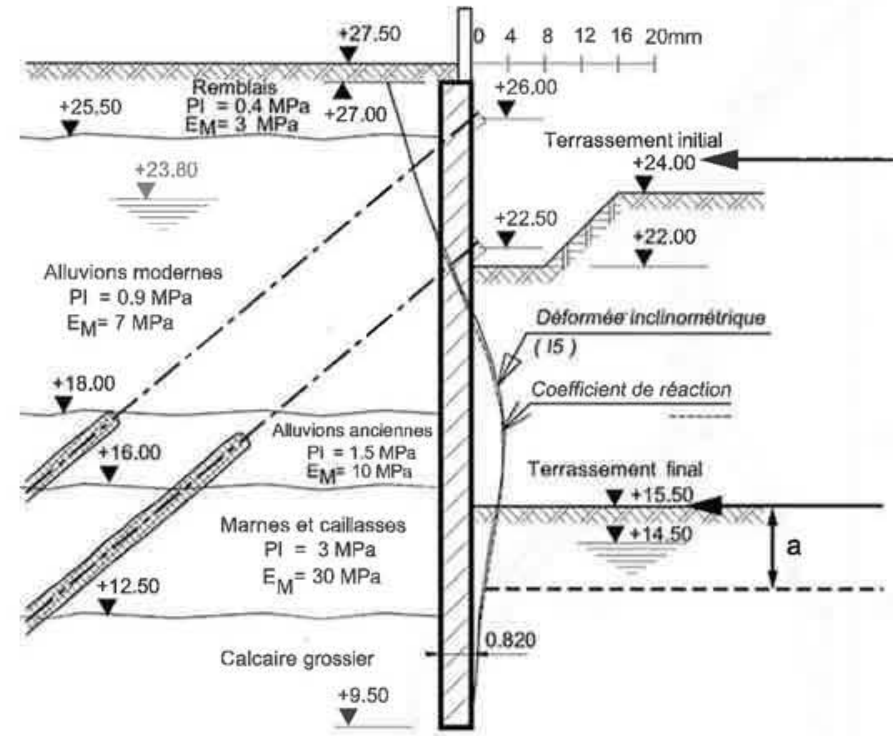

Demi-semelle supérieure sollicitée par la précontrainte des appuis supérieurs

Demi-semelle inférieure sollicitée par la partie supérieure de l'écran

FG. 3 Hauteur d'interaction déduite des mesures inclinométriques. Interaction height derived from inclinometer measurements.

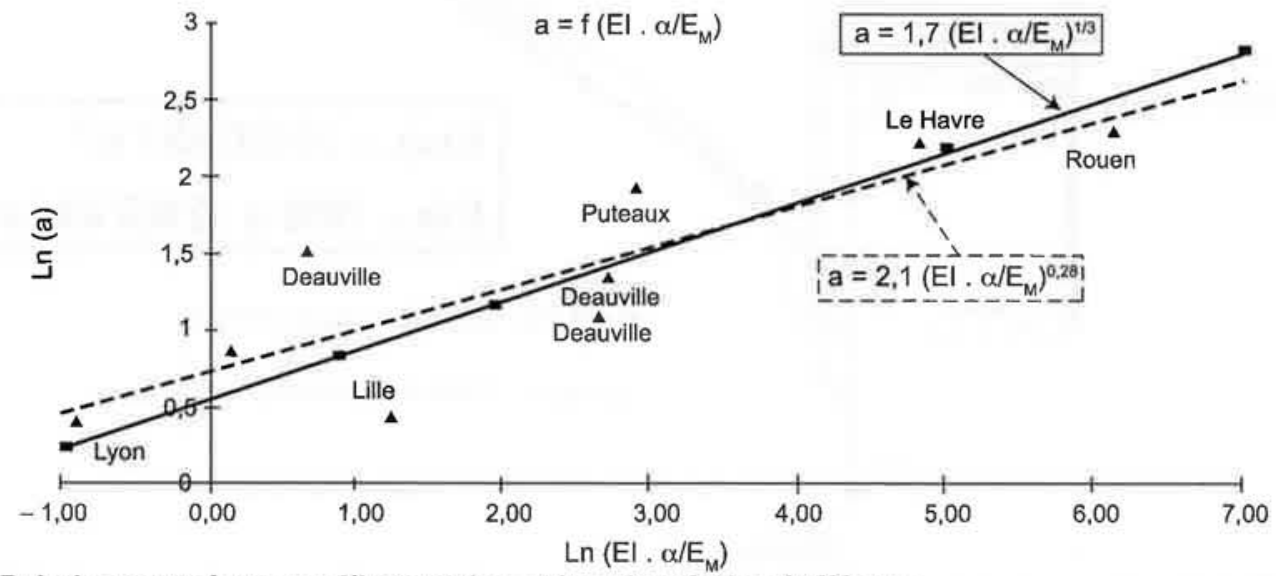

FG. 4 Relation entre hauteur d'interaction et inertie relative de l'écran. Interaction height versus relative wall inertia. 
L'exploitation synthétique des déformées mesurées par inclinométrie a permis d'établir que la hauteur d'influence ( a ) ainsi définie était en moyenne égale à 1,4 fois la longueur élastique $l_{0}$ de l'écran, avec $\mathrm{l}_{0}=(4 . \mathrm{EI} / \mathrm{k})^{1 / 4}$, où EI est le produit d'inertie de l'écran et $\mathrm{k}$ le coefficient de réaction du sol (Schmitt, 1998).

On vérifie en effet que cette expression est compatible avec les formulations empiriques directement issues de l'exploitation des mesures (Schmitt, 1995), soit :

$$
\begin{aligned}
& a=1,7 \cdot\left(E_{I} / E_{M} / \alpha\right)^{1 / 3}(\text { Fig. 4) } \\
& k=3,6 \cdot E_{M} /(\alpha \cdot a)=2,1 \cdot\left(E_{M} / \alpha\right)^{4 / 3} /(E I)^{1 / 3}
\end{aligned}
$$

où $E_{M}$ est le module pressiométrique Ménard et $\alpha$, le coefficient de structure fonction de la nature des sols.

Or l'application directe de la théorie des poutres sur appuis élastiques sollicitées ponctuellement montre que la distance à laquelle le déplacement résiduel n'est plus égal qu'à $20 \%$ du déplacement maximal est précisément égale à $1,6 \times \mathrm{l}_{0}$, à comparer à $1,4 \times \mathrm{l}_{0}$ mesuré (Schmitt, 1998).

Cette concordance à moins de $15 \%$ près entre mesures et prévisions théoriques de l'ordre de grandeur de la hauteur ( a ) démontre clairement le bienfondé de l'application de la théorie des poutres sur appuis élastiques au comportement du sol mobilisé en butée par un écran de soutènement.

Le suivi géotechnique d'une quantité significative d'ouvrages réels depuis le début des années 80 aura donc finalement permis :

- d'établir la validité du modèle « poutre sur appuis élastiques ») pour représenter le comportement réel des ouvrages courants ;

- d'expliquer en partie les raisons de cette validité a priori surprenante ;

- d'établir un ordre de grandeur des valeurs des paramètres empiriques à associer à ce modèle de calcul.

\section{2}

\section{Mise en évidence des limites du modèle " poutre sur appuis élastiques "}

Comme indiqué plus haut, la question à laquelle il devrait être obligatoire de répondre sitôt établie une méthode de dimensionnement ou une formulation de nature empirique est celle de son domaine de validité. Les limites du modèle " poutre sur appuis élastiques »), sur lequel repose actuellement la justification des ouvrages courants, ont pu être cernées à partir de l'observation de quelques ouvrages ayant fait l'objet d'un suivi attentif.

\section{2.}

\section{Limites temporelles}

Le calage des paramètres représentatifs du modèle ( poutre sur appuis élastiques » repose sur le suivi géotechnique d'ouvrages généralement assuré pendant les phases de construction. Malheureusement, on dispose de peu d'exemples de suivi prolongés en phase d'exploitation, ce qui fait que le domaine de validité temporel des paramètres usuels est extrêmement incertain.

L'exemple de la paroi moulée de Colombes fait exception dans la mesure où le suivi de cet ouvrage par inclinométrie a été prolongé pendant plus d'une année après la fin des terrassements, ce qui a permis de mettre en évidence une chute de 1 à 2 des valeurs du coefficient de réaction du sol dans les terrains supérieurs compressibles, constitués de remblais et d'alluvions modernes (Fig. 5 ; Londez et al., 1997).

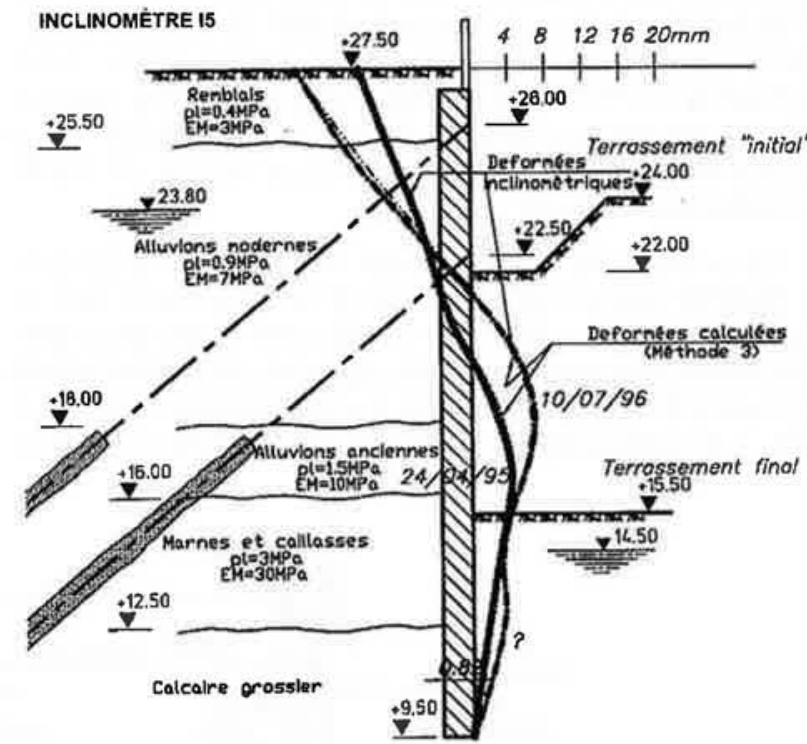

FiG.5 Évolution en 1 an des déplacements de la paroi moulée de Colombes.

Colombes diaphragm wall movements during 1 year.

La généralisation de ce résultat intéressant mais malheureusement ponctuel serait toutefois d'autant plus hasardeuse que :

- on ignore tout de l'évolution ultérieure des déformations de l'ouvrage ;

- l'évolution rapportée ci-dessus concerne essentiellement la partie nord de l'ouvrage, située en bordure de Seine, mais n'a pas été confirmée par le suivi pratiqué dans la partie Sud, sans que cette différence de comportement ait pu être clairement expliquée (variabilité spatiale de la nature des alluvions? comportement d'ensemble du massif limité par les berges de la Seine sous l'effet de la précontrainte ?...) ;

- le cas des alluvions modernes est peut-être particulier. On pourrait être tenté de voir dans l'évolution constatée une simple conséquence de la pratique courante qui consiste à assimiler le module pressiométrique, déduit d'un palier de chargement maintenu pendant une minute, à un module ( à long terme », y compris dans le cas de sols contractants.

Concernant ce dernier point, on rappelle en effet que l'interprétation classique consiste à déduire de l'essai pressiométrique un module d'élasticité drainé en supposant l'identité des modules déviatoriques instantané et différé, identité clairement établie dans le cas d'un matériau dont le comportement serait idéalement élastique et linéaire, ce qui n'est précisément pas le cas des sols.

Au contraire, dans le cas des argiles molles pour lesquelles les déformations différées sont précisément prépondérantes, la contractance génère un comportement non élastique se traduisant par l'apparition de pressions interstitielles y compris dans le domaine déviatorique, d'où il résulte que les déformations mesurées lors de l'essai pressiométrique sont associées à des contraintes effectives plus faibles que ne le sup- 
pose l'interprétation classique, laquelle a donc a priori tendance à surestimer la valeur du module différé.

Les valeurs traditionnelles du coefficient de structure $\alpha$, permettant de passer du module pressiométrique $\mathrm{E}_{\mathrm{M}}$ représentatif du comportement du sol en expansion au module d'Young $\mathrm{E}_{\mathrm{M}} / \alpha$ représentatif du comportement du sol en compression (Gambin, 2005; Ménard, 1965), n'intègrent pas ce phénomène, dont la prise en compte amènerait à envisager des valeurs de $\alpha$ croissantes quand le degré de consolidation de l'argile diminue, alors que c'est l'inverse qui est traditionnellement retenu.

Un coefficient d'ajustement théorique à appliquer au module pressiométrique pour tenir compte de ces phénomènes, fonction du coefficient A proposé par Skempton pour exprimer, précisément, les surpressions interstitielles générées par des sollicitations déviatoriques, a pu être proposé par Lavisse et Schmitt (2004), et

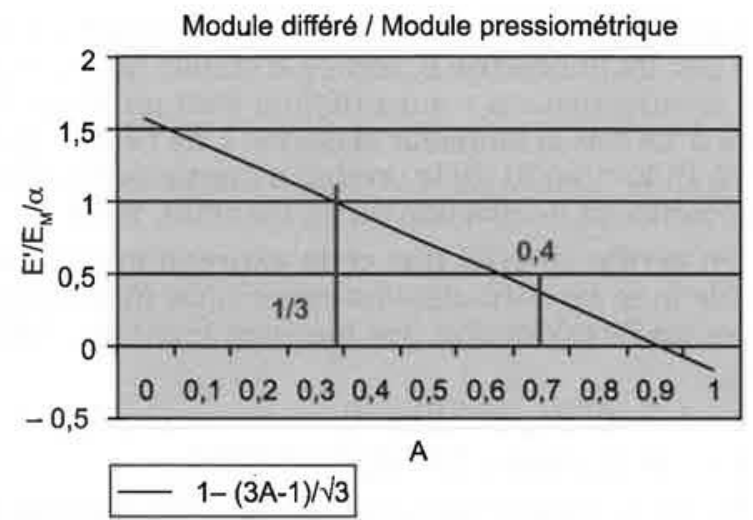

FG.6 Rapport entre module différé et module pressiométrique en fonction du coefficient $\mathrm{A}$ de Skempton.

Ratio between long term and pressuremeter moduli versus Skempton A coefficient.

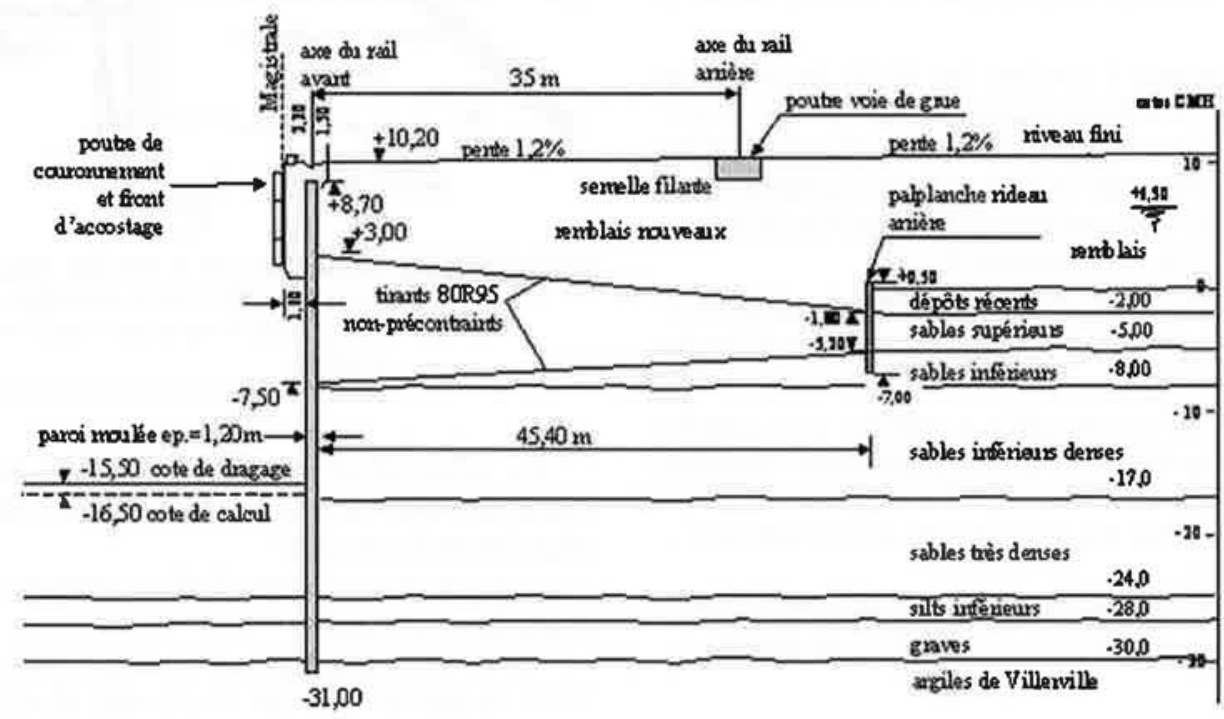

ตG. 7 Mur de quai de Port 2000 au Havre.

Le Havre Port 2000 quay wall.

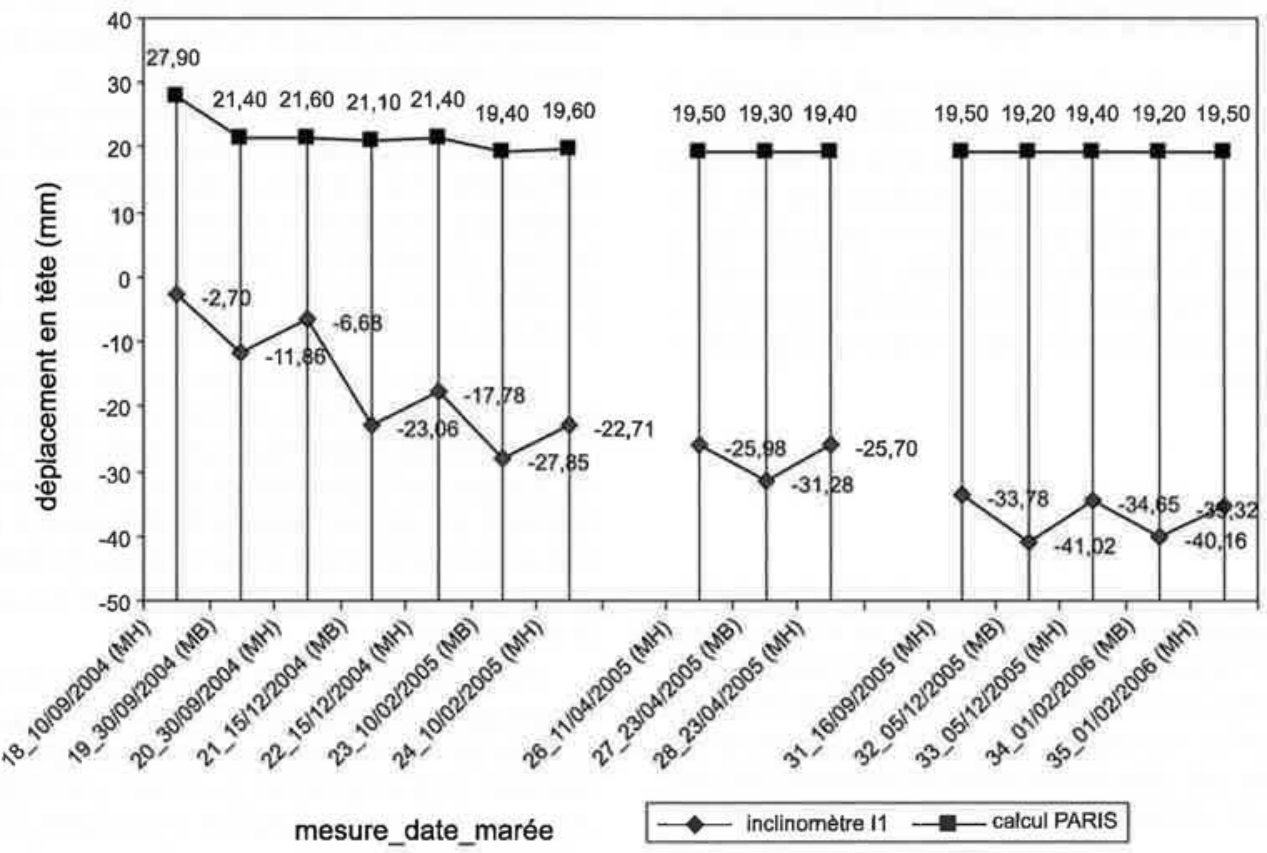

n๑.8 Déplacements calculés et mesurés au Port du Havre en fonction du temps et des cycles de marées. Calculated and measured displacements in Le Havre versus time and tide cycles. 

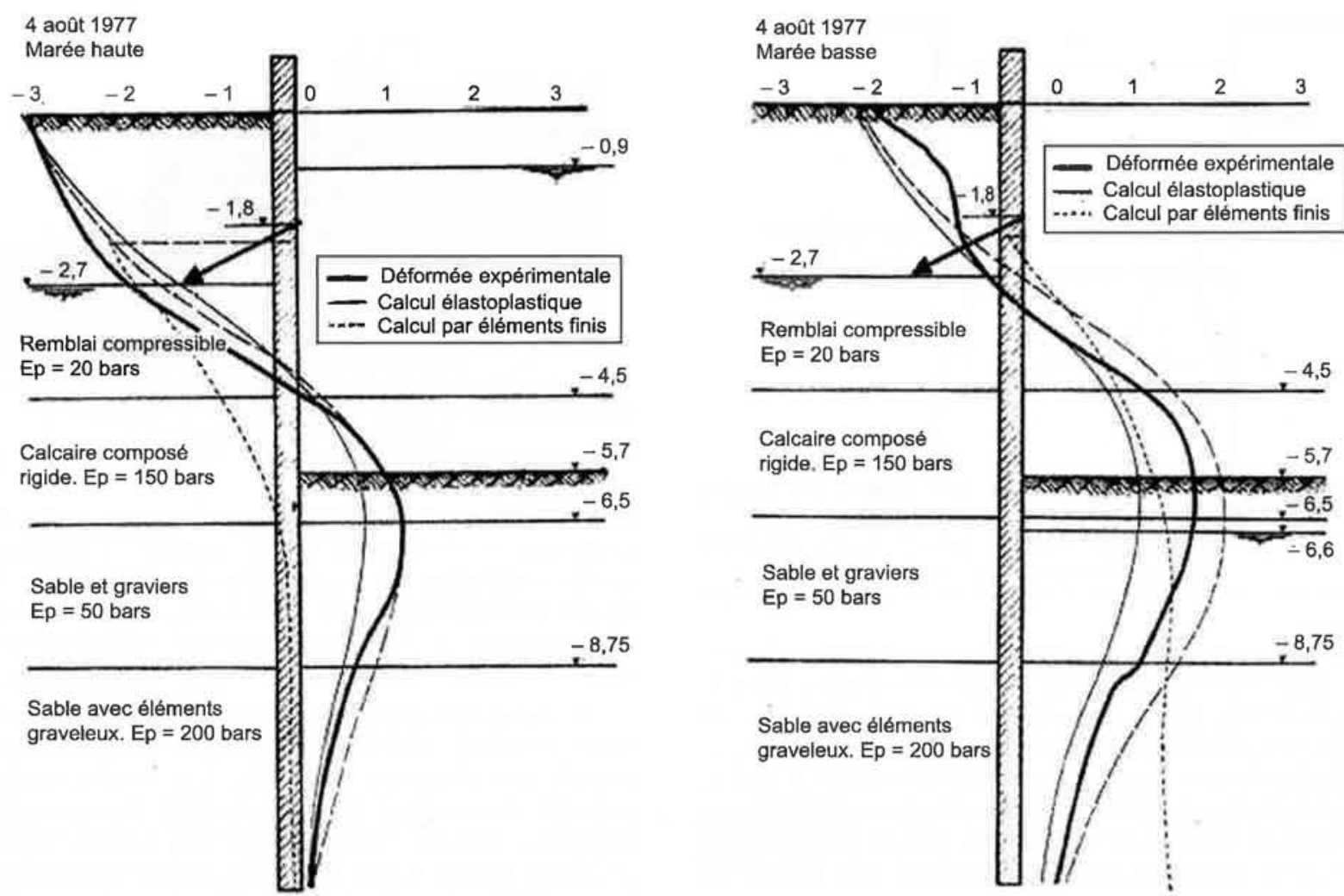

nG.9 Déplacements calculés et mesurés du mur de quai de Deauville en fonction des cycles de marées $\left(1 \mathrm{bar}=10^{5} \mathrm{~Pa}\right)$.

Calculated and measured displacements of Deauville quay wall during tide cycles.

corroboré par le suivi géotechnique consistant à mesurer le tassement en fonction du temps sous des remblais de préchargement au port de Rouen (Fig. 6).

Il va sans dire que d'autres exemples de tels suivis seraient nécessaires pour préciser de façon plus systématique les valeurs représentatives du comportement à long terme des argiles, en l'absence d'essais oedométriques.

Un autre exemple de méconnaissance des effets du temps est celui des ouvrages maritimes soumis à des sollicitations cycliques : la section instrumentée de la paroi moulée du quai de Port 2000 au Havre met ainsi en évidence l'effet des cycles de marée sur les déplacements en tête, notablement différents des prévisions données par les calculs traditionnels, que ce soit en termes d'amplitude ou en termes d'évolution (Figs. 7 et 8 ; Delattre et al., 2005 ; Marten, 2005 ; Sayavong, 2008).

Inversement, le comportement du mur de quai de Deauville en paroi préfabriquée, continûment observé au cours des cycles de marées avait pu, quant à lui, être correctement prédit par les calculs classiques (Fig. 9 ; Bustamante et Gouvenot, 1978).

On verra qu'une explication possible de cette différence peut être trouvée dans un effet d'échelle, puisqu'il s'agit d'un ouvrage de 1,2 m d'épaisseur et de $40 \mathrm{~m}$ de hauteur dans le premier cas, de 0,35 m d'épaisseur et de $12 \mathrm{~m}$ de hauteur dans le second.

On met ainsi en évidence un autre problème, qui est celui de la validité des modèles de comportement traditionnels dans le domaine non plus temporel, mais spatial.

\section{2 .2}

\section{Limites géométriques}

Il est clair que le modèle de comportement « poutres sur appuis élastiques »), dont la validité a été démontrée vis-à-vis de la prédiction du comportement à court terme des ouvrages courants isolés, peut difficilement prétendre à la même efficacité vis-à-vis de la prise en compte d'interactions entre ouvrages voisins, ou de la prise en compte de la déformation d'ensemble du massif de sol avoisinant l'écran.

La difficulté pratique à laquelle doit faire face le concepteur est donc de déceler l'existence de ces phénomènes, et d'apprécier à partir de quand il y a lieu de les prendre explicitement en compte au moyen d'une méthode appropriée. On conçoit que le suivi géotechnique joue ici encore un rôle déterminant.

Un exemple de circonstance dans laquelle l'interaction entre ouvrages voisins peut jouer un rôle favorable vis-à-vis du comportement de l'écran est celui des tranchées couvertes de faible largeur, dans lesquelles les ( bulbes de contraintes » correspondant à la mobilisation du terrain en butée sous le niveau de l'excavation ont naturellement tendance à interagir.

Un tel exemple a pu être donné par le suivi géotechnique de la paroi moulée de la trémie Pasteur, à Rouen, dont les déformations mesurées se sont trouvées être environ quatre fois plus faibles que prévu. L'explication a pu en être partiellement trouvée dans un effet tridimensionnel, lié d'une part à la localisation de la section de mesures au voisinage d'une transition entre deux zones appuyées différemment et, d'autre part, au mode de butonnage progressif en fonction de l'avancement de l'excavation, non pris en compte par les calculs à deux dimensions traditionnels (Nguyen, 2003).

Cependant un coefficient de réaction trois fois plus élevé que celui résultant de la formulation empirique rappelée au § 3.1, et établie à partir du suivi d'ouvrages isolés, a pu être justifié dans ce cas en estimant tout simplement le raccourcissement élastique de la tranche de sol comprimé par les deux parois en vis-à-vis, ce qui est relativement logique (Fig. 10 ; Escobar, 2001). 


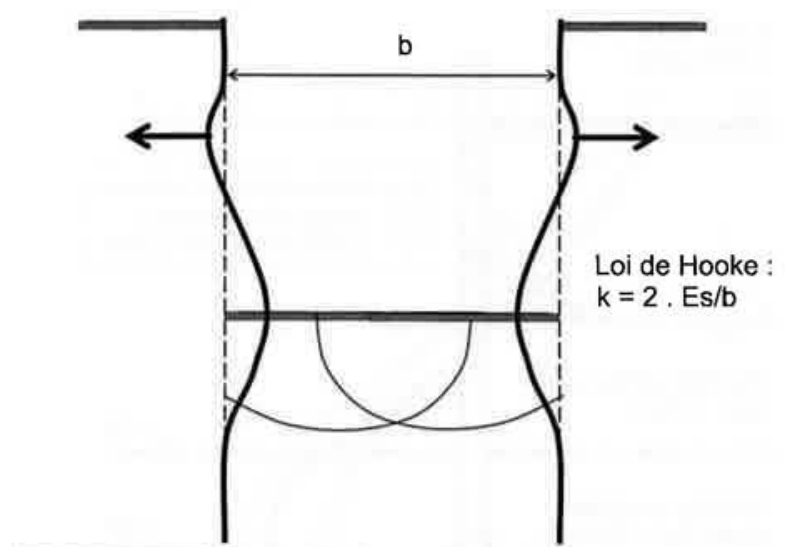

Fi6.10 Interaction entre les zones de butée mobilisées par deux écrans rapprochés. Interaction between passive earth pressure areas mobilized by close together walls.

Un autre exemple classique d'interaction, défavorable cette fois-ci, est celui de tirants scellés au terrain à une trop faible distance de l'écran pour que les sollicitations induites dans le massif amont puissent être dissipées par cisaillement interne : c'est ce qui se produit lorsque la proximité d'ouvrages enterrés, ou l'interdiction de réaliser des tirants sous le tréfonds des immeubles, ne permet pas de satisfaire à la condition de Kranz.

Dans ce cas, la mise en butée du massif par les scellements induit un complément d'efforts sur l'écran que ne permettent pas de prendre en compte les méthodes classiques d'évaluation de la poussée des terres, et qu'il y a lieu d'intégrer au moyen de méthodes appropriées.

Tel est le cas par exemple de l'écran composite (paroi berlinoise surmontant une paroi moulée) réalisé à Monaco pour la construction du parking semienterré de l'immeuble Le Testimonio (Fig. 11 ; Schlosser, 2007) : l'interdiction administrative de réaliser des tirants de plus de $15 \mathrm{~m}$ de longueur rendait à l'évidence

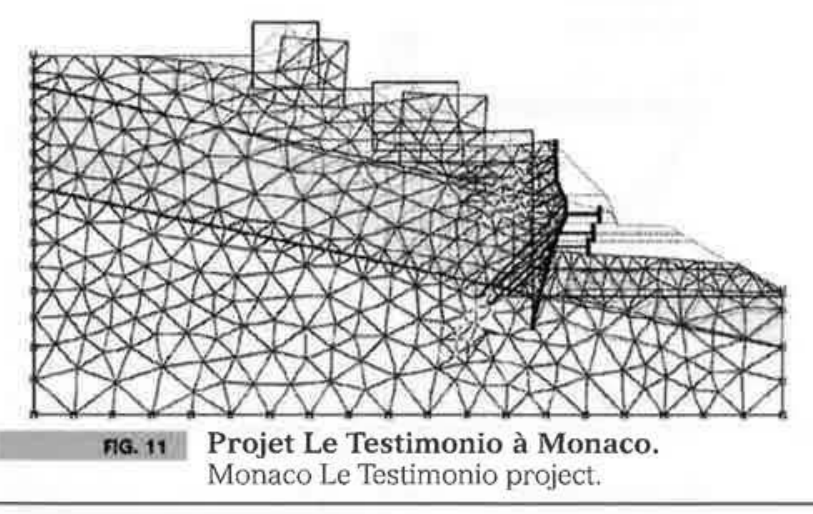

leur efficacité problématique vis-à-vis de la réalisation d'une excavation de $35 \mathrm{~m}$ de hauteur, le supplément d'efforts résultant de l'inévitable interaction étant alors repris par les planchers de la superstructure, dont la construction devait alors impérativement être commencée avant les phases d'excavation profondes.

Le suivi géotechnique a dans ce cas fort heureusement confirmé l'ordre de grandeur des résultats des calculs aux éléments finis (Fig. 12), seuls capables de prendre en compte non seulement les sollicitations supplémentaires induites par les tirants trop rapprochés, mais encore le déplacement d'ensemble du massif d'éboulis dans lequel étaient scellés ces tirants, déplacement ignoré par les calculs traditionnels qui supposent fixes les points d'ancrage, et n'intègrent donc dans la déformée calculée que l'effet de l'allongement élastique du tirant.

Cet exemple réel met bien en évidence le grand intérêt de la méthode de Kranz en tant qu'indicateur des limites de validité des méthodes de calcul traditionnelles.

Cependant, une question encore mal résolue est celle, souvent associée à la précédente mais pas nécessairement, de la limite à partir de laquelle il convient de prendre en compte le risque d'un déplacement d'en-

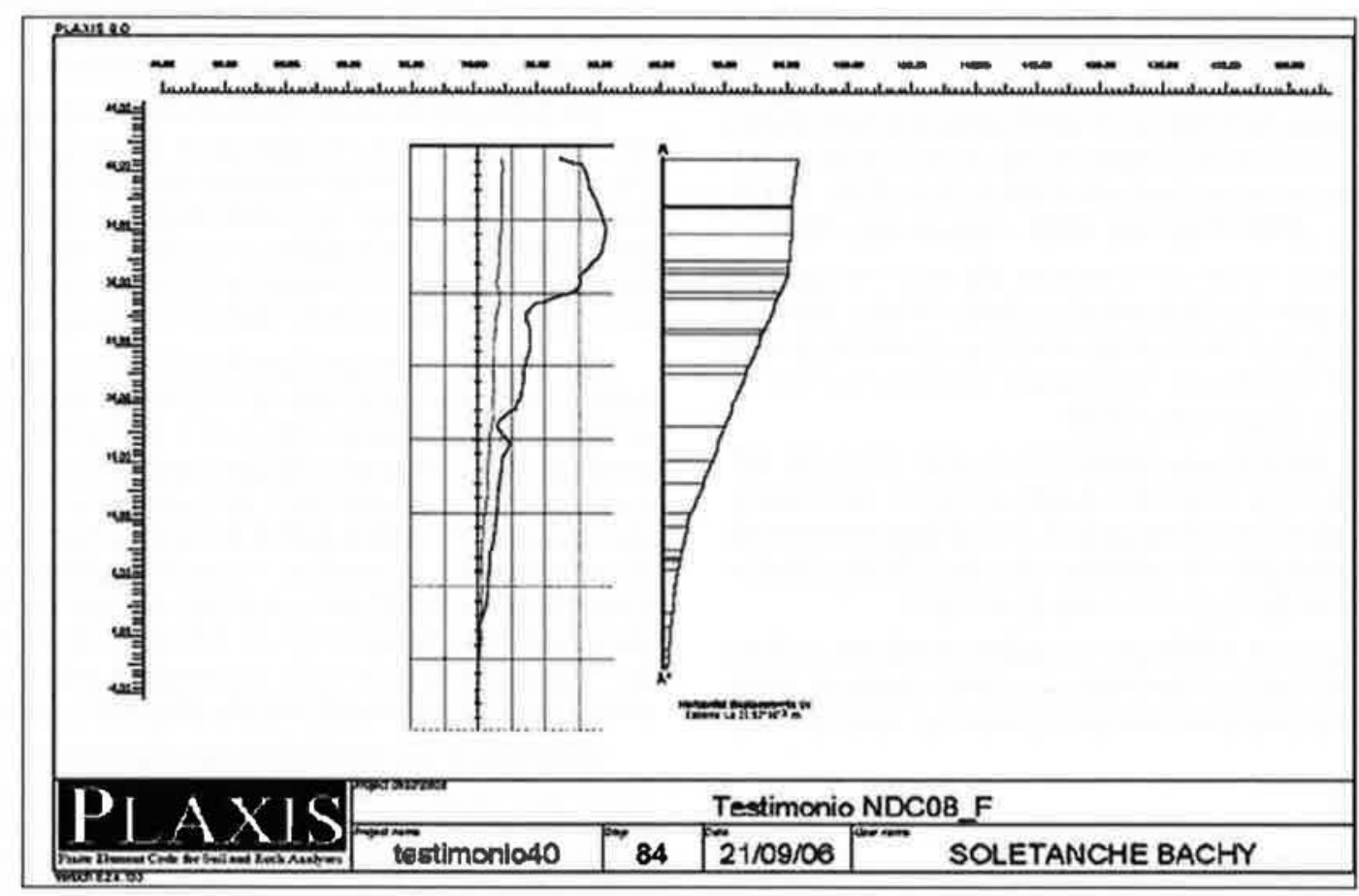




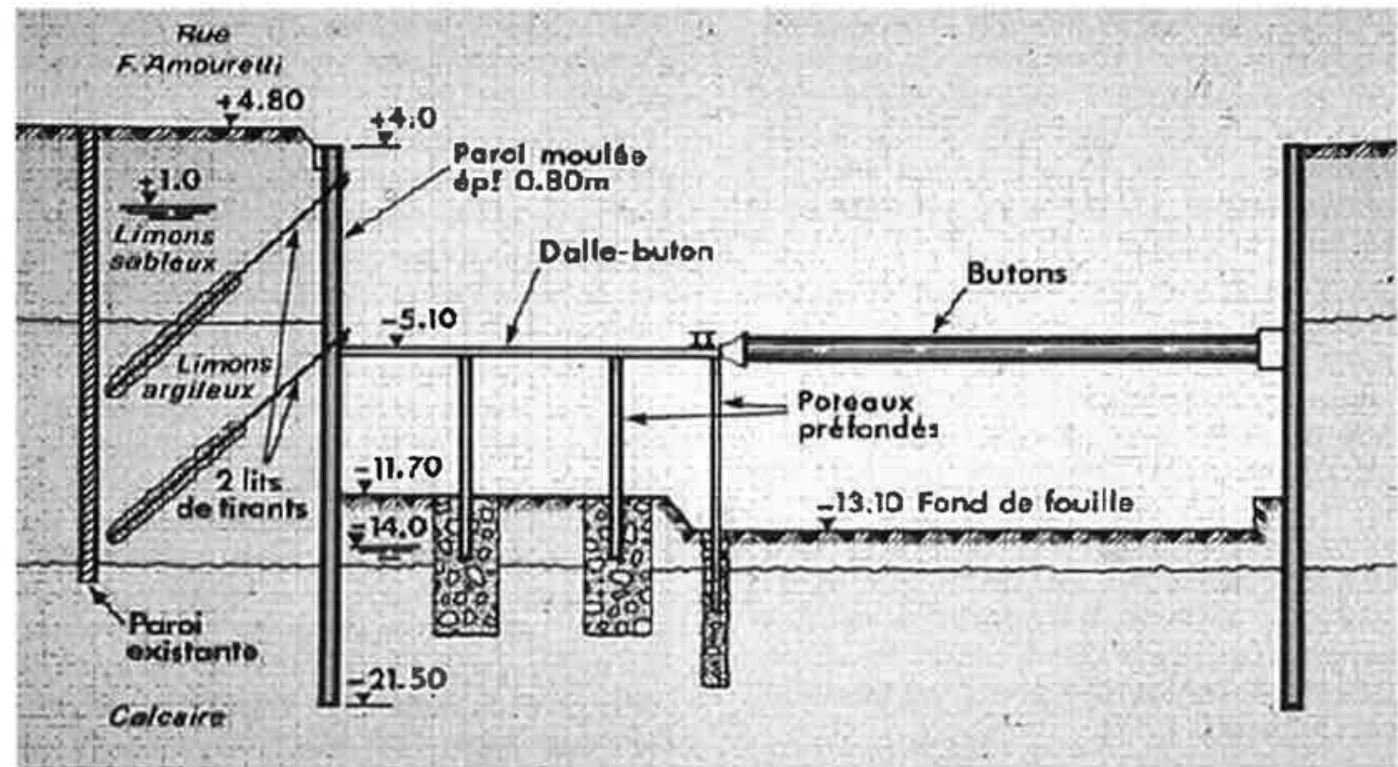

FG. 13 Dispositif de soutènement de la fouille de l'hôtel Hilton à Cannes.

Retaining structure for Cannes Hilton hotel excavation.

semble important du massif de sol, sans que ce déplacement ne soit nécessairement associé à une interaction entre les ancrages et l'écran.

Ainsi l'exemple du Testimonio est particulièrement intéressant dans la mesure où se posaient clairement les deux problèmes (interaction et déplacement d'ensemble).

Le cas plus ancien de la paroi moulée du parking de l'hôtel Hilton, à Cannes (Fig. 13), donne l'exemple d'une interaction liée à l'impossibilité de réaliser des tirants assez longs compte tenu de l'existence d'une paroi adjacente, interaction encore une fois décelée au moyen de la méthode de Kranz et prise en compte, non pas par la méthode des éléments finis, mais par ajout manuel à la courbe de poussée du supplément provenant de la part de réaction d'appui non dissipée dans le massif amont, et repris par butonnage complémentaire (butonnage intérieur lui-même insuffisant pour reprendre la réaction totale compte tenu des portées importantes et des risques de flambement associés, raison de ce système d'appui composite dans lequel les tirants étaient davantage considérés comme un frein que comme un appui à part entière).

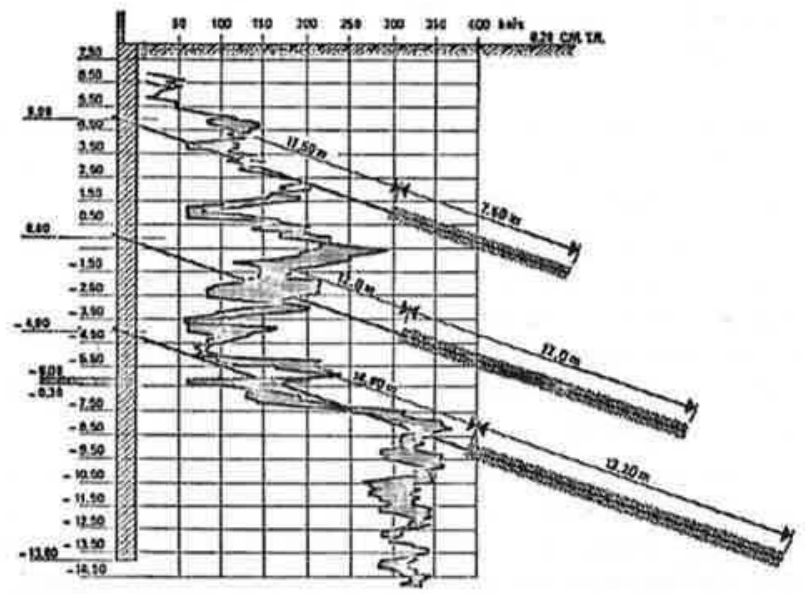

nG.14 Paroi moulée et ancrages permanents de la centrale de Gravelines.

Gravelines power plant permanent diaphragm wall and anchors.
Dans ce cas, le problème posé, et ainsi résolu, était celui d'une interaction et non d'un déplacement d'ensemble, lequel n'a d'ailleurs nullement été mis en évidence.

A l'inverse, l'exemple encore plus ancien mais désormais classique de la paroi moulée du canal d'amenée de la centrale de Gravelines est celui de tirants définitifs scellés dans un massif de sables limoneux, dont le suivi géotechnique a mis en évidence le déplacement d'ensemble, notablement supérieur au déplacement calculé, lequel n'intégrait que les déformations locales de l'écran lui-même et l'allongement élastique du tirant (Figs. 14 et 15; Bustamante et Gouvenot, 1978).

Dans ce cas, l'absence d'interaction avait pu être vérifiée par le respect de la condition de Kranz, et le problème posé est donc exclusivement celui de la déformation intrinsèque du massif arrière sollicité, sur l'ensemble de la hauteur de l'écran, par plusieurs niveaux de tirants d'ancrage, ce qu'il avait à l'époque été convenu d'appeler ( effet-gabion » par analogie avec le fonctionnement des ouvrages massifs réalisés au moyen de gabions de palplanches métalliques.

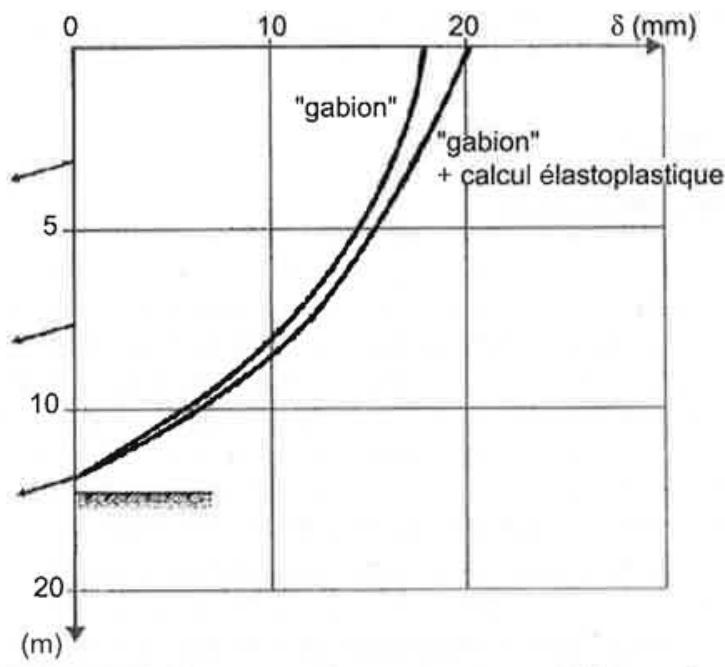

Fic. 15 Comparaison entre déformée et déplacement d'ensemble de la paroi de Gravelines.

Comparison between Gravelines diaphragm wall deflection and overall displacement. 
Il semble donc bien que le modèle de comportement ( effet gabion », ou " déformation du massif de sol sollicité par un soutènement multi-ancrages », soit à distinguer, même s'il peut dans certains cas lui être superposé, du modèle « interaction entre les ancrages et l'écran » généralement associé au non respect de la condition de Kranz.

30 ans après l'exemple de Gravelines, on ne dispose toujours pas d'indicateur permettant de fixer clairement les limites du déclenchement de ce modèle de comportement particulier, indicateur à rechercher probablement non pas dans la résistance au cisaillement du sol comme dans le cas de Kranz mais dans les paramètres de déformation du sol (l'ordre de grandeur des déplacements mesurés à Gravelines avait à l'époque pu être parfaitement retrouvé par simple intégration des équations de Bresse, permettant d'approcher la déformée élastique, principalement due à l'effort tranchant, de la « poutre de sol » constituée par le gabion fictif; Bustamante et Gouvenot, 1978).

Il est à cet égard intéressant de comparer les exemples de Colombes et de Gravelines (Figs. 3 et 14), dans la mesure où il s'agit dans les deux cas de soutènements multi-ancrages permanents, dont les suivis géotechniques à un ou deux ans ont mis en évidence l'absence totale de déplacement d'ensemble dans un cas (le modèle ( poutre sur appuis élastiques ) s'étant au contraire avéré d'une pertinence remarquable dans l'exemple de Colombes), l'existence d'un déplacement d'ensemble non seulement significatif, mais encore prépondérant dans l'autre cas.

L'explication de ces deux comportements extrêmes se trouve à l'évidence dans la comparaison des modules de déformation des massifs d'ancrage des scellements : marnes et caillasses de la région parisienne dans un cas, sables limoneux de Dunkerque dans l'autre.

Un autre intérêt majeur du suivi pratiqué à Gravelines aura été la mise en évidence du temps important, de l'ordre d'une année, mis par l'effet-gabion à se manifester. Ceci renvoie au paragraphe précédent (limites temporelles des modèles de comportement), et remet en cause la pratique générale qui consiste à ne s'inquiéter de l'évolution des déformations dans le temps que dans le cas des terrains argileux, réputés susceptibles de fluer alors que les massifs sableux ne le seraient pas..

\section{2 .3}

\section{Limites mécaniques}

On a vu que la pertinence du modèle « poutre sur appuis élastiques ») avait pu être démontrée vis-à-vis de la représentation des modes de sollicitations usuels des ouvrages les plus courants, à savoir la mise en butée du sol côté amont sous l'effet des charges concentrées exercés par les appuis supérieurs, d'une part, la mise en butée du sol constituant l'appui inférieur de l'écran sous le niveau du fond de fouille, d'autre part.

On a vu également que l'identité des coefficients de réaction associés à ces deux modes de sollicitations classiques s'expliquait par le fait que la « hauteur d'influence ») du chargement était dans les deux cas fonction du même paramètre, à savoir la rigidité relative de l'écran par rapport au sol, traduite par la notion classique de ( longueur élastique ), $1_{0}$ (comme l'avait d'ailleurs déjà mis en évidence Westergaard
(1926) dans le domaine du comportement des radiers soumis aux sollicitations ponctuelles exercées par des poteaux).

Le suivi géotechnique d'ouvrages réels a également permis de mettre en évidence d'autres modes de fonctionnement, ne remettant pas nécessairement en cause la validité pratique du modèle «poutre sur appuis élastiques »), mais démontrant la nécessité d'associer à ces modes de sollicitation spécifiques des valeurs particulières du coefficient de réaction.

Ainsi on observe parfois, dans le cas d'écrans de soutènement très rigides réalisés dans des terrains très compressibles (Fig. 16 par exemple), des déplacements plus faibles que ceux évalués au moyen de la formulation précédemment rappelée : cela s'explique parfaitement dans la mesure où la hauteur d'interaction, dont on a vu que l'ordre de grandeur prévu par la théorie des poutres sur appuis élastiques et confirmé par le suivi géotechnique d'ouvrages réels était $1,5 \times 1_{\alpha}$ peut être alors supérieure à la fiche réelle de l'écran, ce qui n'a physiquement aucun sens.

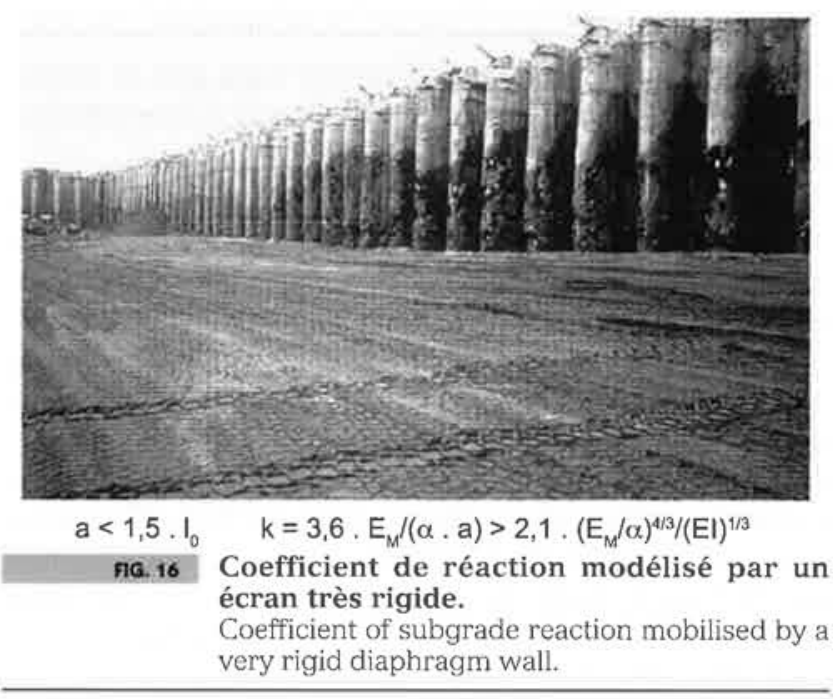

Il y a donc lieu en pareil cas, comme cela était d'ailleurs recommandé dès l'origine (Schmitt, 1995), d'utiliser la formule d'évaluation du coefficient de réaction sous sa forme décomposée en distinguant :

- la hauteur d'interaction, qu'il convient alors de borner aux 2/3 de la fiche totale comme initialement recommandé par Terzaghi et Ménard;

- le coefficient de réaction, proportionnel au module de déformation du sol et inversement proportionnel à la hauteur d'interaction ( a ), et dont la valeur moyenne a pu empiriquement être approchée par l'expression générale : $k=3,6 \cdot E_{M} /(\alpha . a)$.

A l'inverse, on est parfois surpris d'observer, à l'occasion d'essais de rabattement de grande ampleur réalisés à l'intérieur d'une enceinte périmétrale étanche, des déplacements notablement supérieurs à ceux calculés en utilisant des valeurs traditionnelles du coefficient de réaction.

C'est oublier que le mode de sollicitation de l'écran n'a alors plus rien à voir avec le cas d'un chargement ponctuel, puisqu'il s'agit au contraire de l'application d'un différentiel de pression hydrostatique, triangulaire sur la hauteur du rabattement et uniforme en partie inférieure, appliqué à l'ensemble de la hauteur de l'écran : la hauteur d'interaction n'a alors à l'évidence plus rien à voir avec l'inertie de l'écran, dont le compor- 


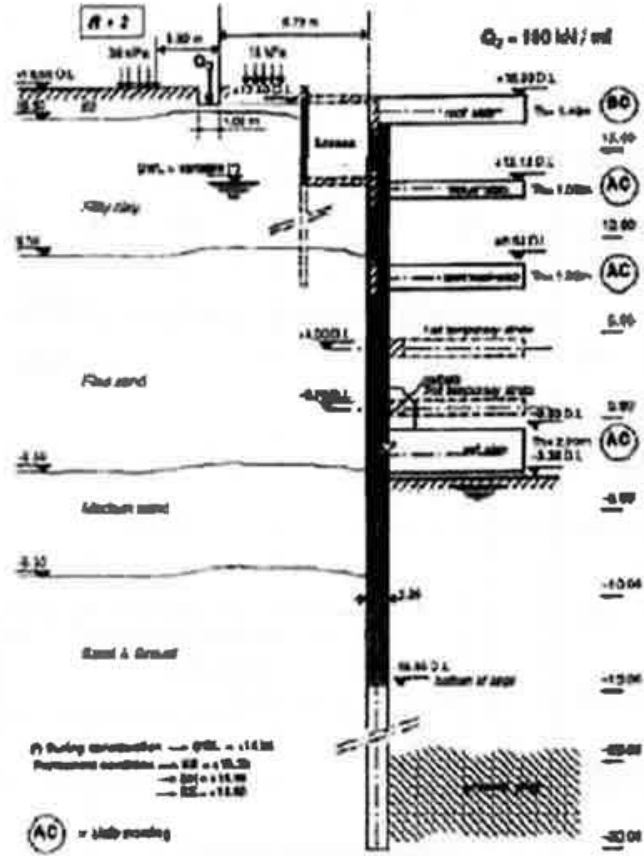

FG. 17 Paroi moulée de la station El Behoos du métro du Caire.

El Behoos station diaphragm wall, Cairo metro project.

\section{Déplacement (mm)}

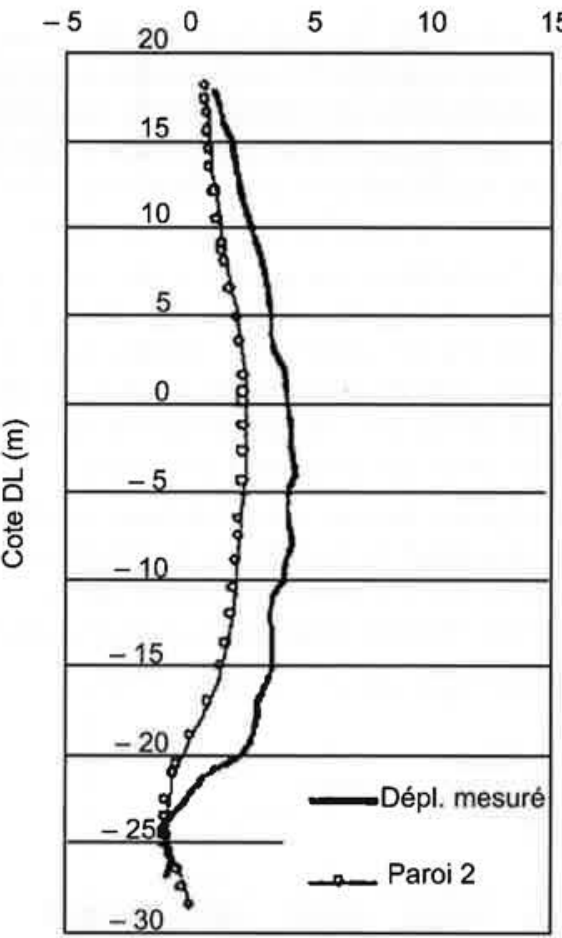

F16. 18 Comparaison entre déplacements calculé et mesuré de la paroi moulée de la station El Behoos pendant l'essai de pompage.

Comparison between El Behoos diaphragm wall calculated and measured displacements during the pumping test.

tement s'apparente plutôt à celui d'une semelle souple de grandes dimensions uniformément chargée.

Le coefficient de réaction devrait alors en pareil cas être évalué, comme précédemment, en utilisant une valeur de a plus proche de la hauteur totale de la fiche que de la longueur élastique de l'écran, laquelle peut s'avérer cette fois-ci notablement plus faible.

C'est ce qu'a mis clairement en évidence le suivi géotechnique des déformations de la paroi moulée de la station El Behoos de la ligne 2 du métro du Caire (Fig. 17 ; Bazin et Schmitt, 2001), au moyen d'inclinomètres de près de $50 \mathrm{~m}$ de hauteur ayant permis de distinguer l'effet des terrassements, à $25 \mathrm{~m}$ de profondeur, et celui du rabattement, qui avait fait l'objet d'un essai préalable au cours duquel il a été possible d'observer une translation de la paroi moulée, uniquement limitée en pied par l'encastrement dans le radier injecté réalisé entre 40 et $47 \mathrm{~m}$ de profondeur (Fig. 18).

La difficulté pratique est la superposition, pendant les phases de terrassement, des modes de sollicitations mécaniques (mise en butée du sol par recherche d'un appui inférieur) et hydrauliques (entrainement de la fiche sous l'effet de la poussée hydrostatique), superposition vis-à-vis de laquelle on peut supposer que la valeur pertinente du coefficient de réaction est intermédiaire entre les valeurs associées à ces deux modes de sollicitation, sans qu'il soit malheureusement possible d'en dire beaucoup plus (Fig. 19).

Se pose également de façon cruciale le problème de la limite à partir de laquelle ce modèle de comportement ( fiche hydraulique ») devient déterminant : il a pu être mis en évidence au Caire compte tenu de l'importance exceptionnelle de l'ouvrage et des sollicitations associées, sans être pour autant critique compte tenu de la compacité des terrains.

Il peut être négligé dans les cas courants, comme le montre clairement le suivi géotechnique généralement pratiqué sur ces ouvrages, mais peut au contraire redevenir critique dans le cas d'ouvrages qui, sans être de dimensions exceptionnelles, seraient réalisés dans des terrains fortement compressibles.

L'établissement d'indicateurs permettant de préciser les limites d'apparition de ce modèle de comportement reste un sujet de recherche appliquée particulièrement important.

On a ainsi pu mettre en évidence la pertinence du modèle "fiche hydraulique » dans le cas du mur de quai du Havre (Figs. 8 et 20 ; Delattre et al., 2005; Marten, 2005; Sayavong, 2008), où l'effet de la marée, même de faible amplitude, se traduisait par un déplacement d'ensemble significatif sur la totalité de la hauteur de l'ouvrage (dont on rappelle qu'elle atteint $40 \mathrm{~m}$, ce qui fait qu'une marée de faible amplitude se traduit par

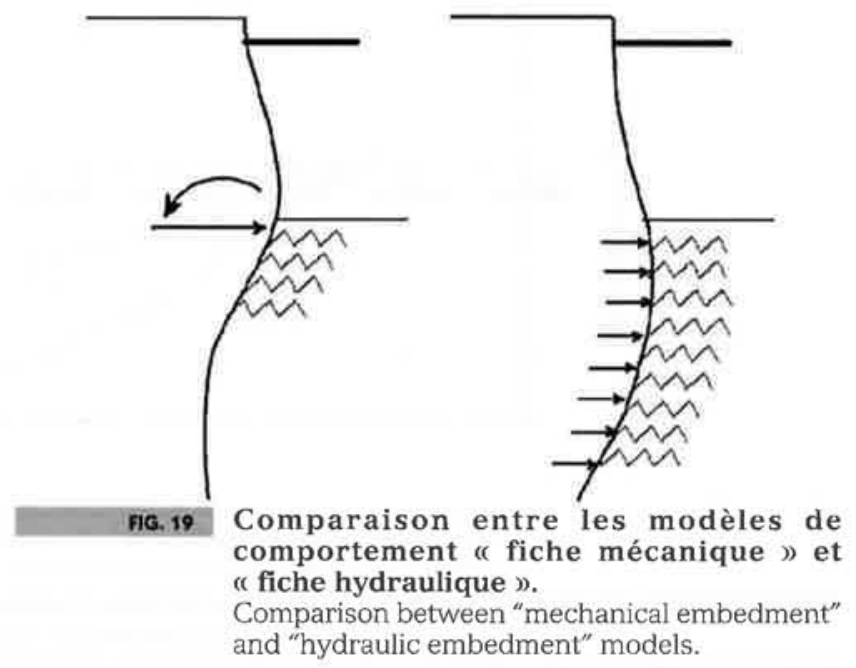




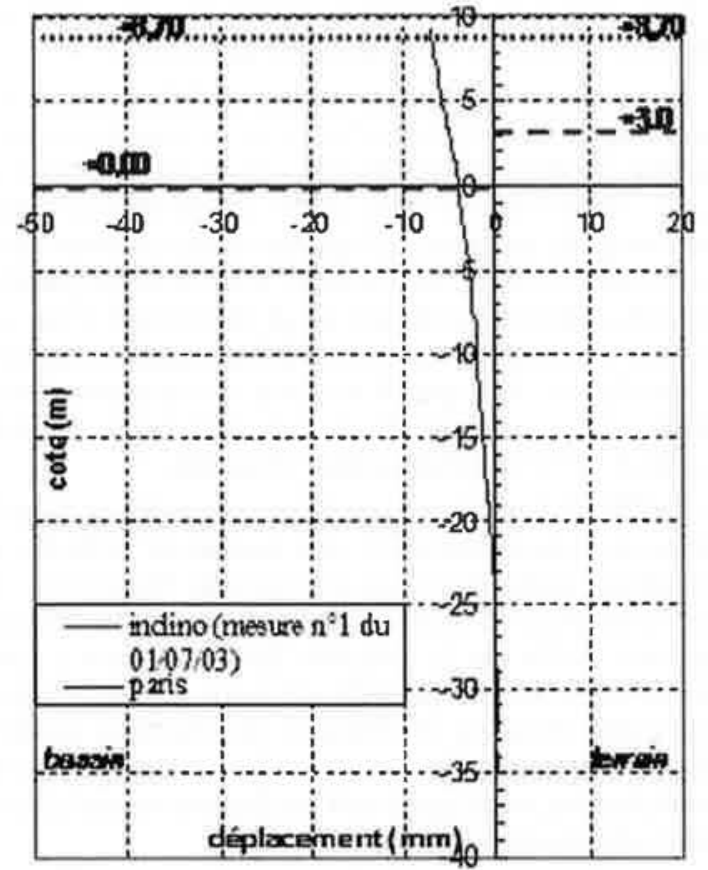

FiG.20 Comparaison entre déplacements calculés et mesurés du mur de quai du Havre lors d'un rabattement sans excavation.

Comparison between Le Havre quay wall calculated and measured displacements while pumping without excavating.

une sollicitation résultante considérable), et finalement par un comportement du sol plus souple que prévu, entraînant à la fois des déplacements plus importants et des courbures plus faibles (Figs. 21 et 22).

A l'inverse, il a pu être légitimement négligé dans le cas du mur de quai de Deauville, dont on rappelle que la hauteur n'excède pas 12 m (Figs. 9 et 23).

Sur le plan mécanique, une autre limitation du modèle ( poutre sur appuis élastiques » est son incapacité à prendre en compte les arcs de décharge, qui peuvent exister aussi bien dans des plans horizontaux, dans le cas d'excavations de petites dimensions, que dans le plan vertical.

Il ne s'agit d'ailleurs pas à proprement parler, là non plus, d'une limitation de la méthode, dans la mesure où les programmes de calcul permettent souvent l'in-

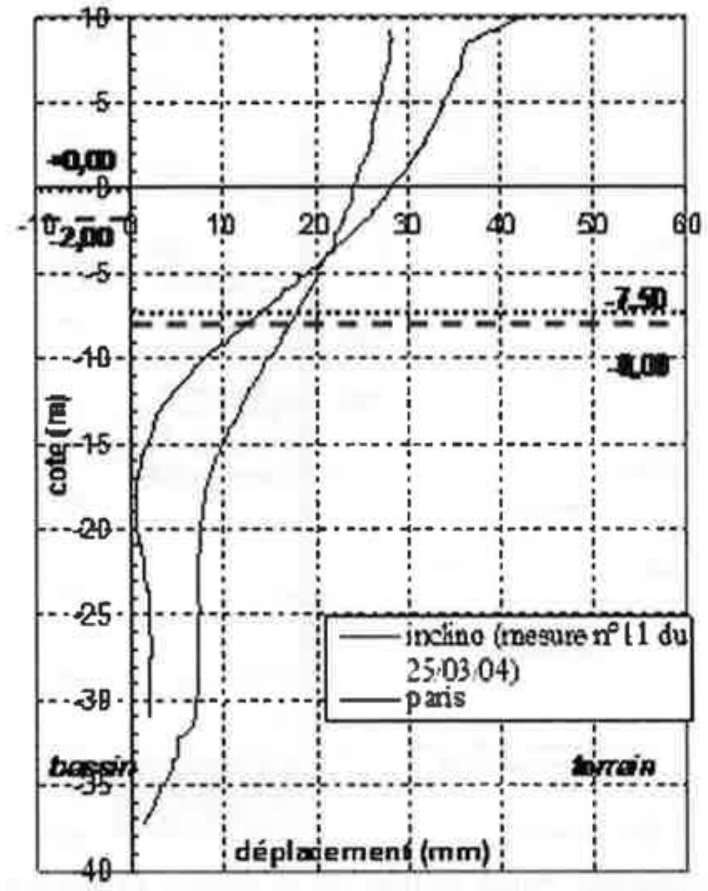

F6.21 Comparaison entre déformées calculées et mesurées du mur de quai du Havre lors d'une phase d'excavation.

Comparison between Le Havre quay wall calculated and measured deflections during an excavation stage.

troduction manuelle de diagrammes de poussée (diagrammes empiriques de Terzaghi et Peck par exemple), certains programmes d'origine anglo-saxonne proposant même des algorithmes permettant une prise en compte automatique des transferts de charges des zones déformables vers les zones plus rigides.

De tels transferts ont pu être mis en évidence à l'occasion du suivi de plusieurs ouvrages; leur importance relative est d'autant plus grande que la hauteur excavée et la hauteur d'eau sont faibles, ce qui justifie parfaitement qu'on les considère généralement comme négligeables pour les ouvrages courants.

Le suivi géotechnique de quelques ouvrages particuliers a cependant pu mettre en évidence, dans le cas d'excavations hors d'eau, un risque de sous-estimation des sollicitations pouvant s'avérer critique dans

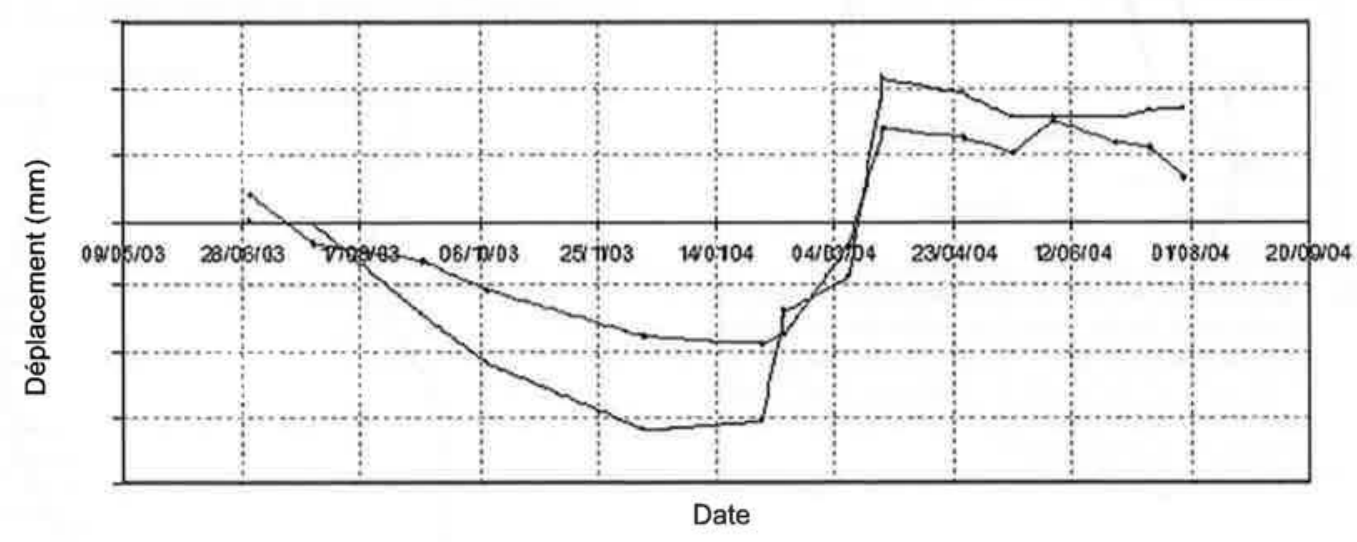

$\rightarrow$ Mesures inclinométriques $\quad-$ Calcul Paris avec hypothèses chantier

F6. 22 Évolution des déplacements calculés et mesurés du mur de quai du Havre. Le Havre quay wall calculated and measured displacements versus time. 

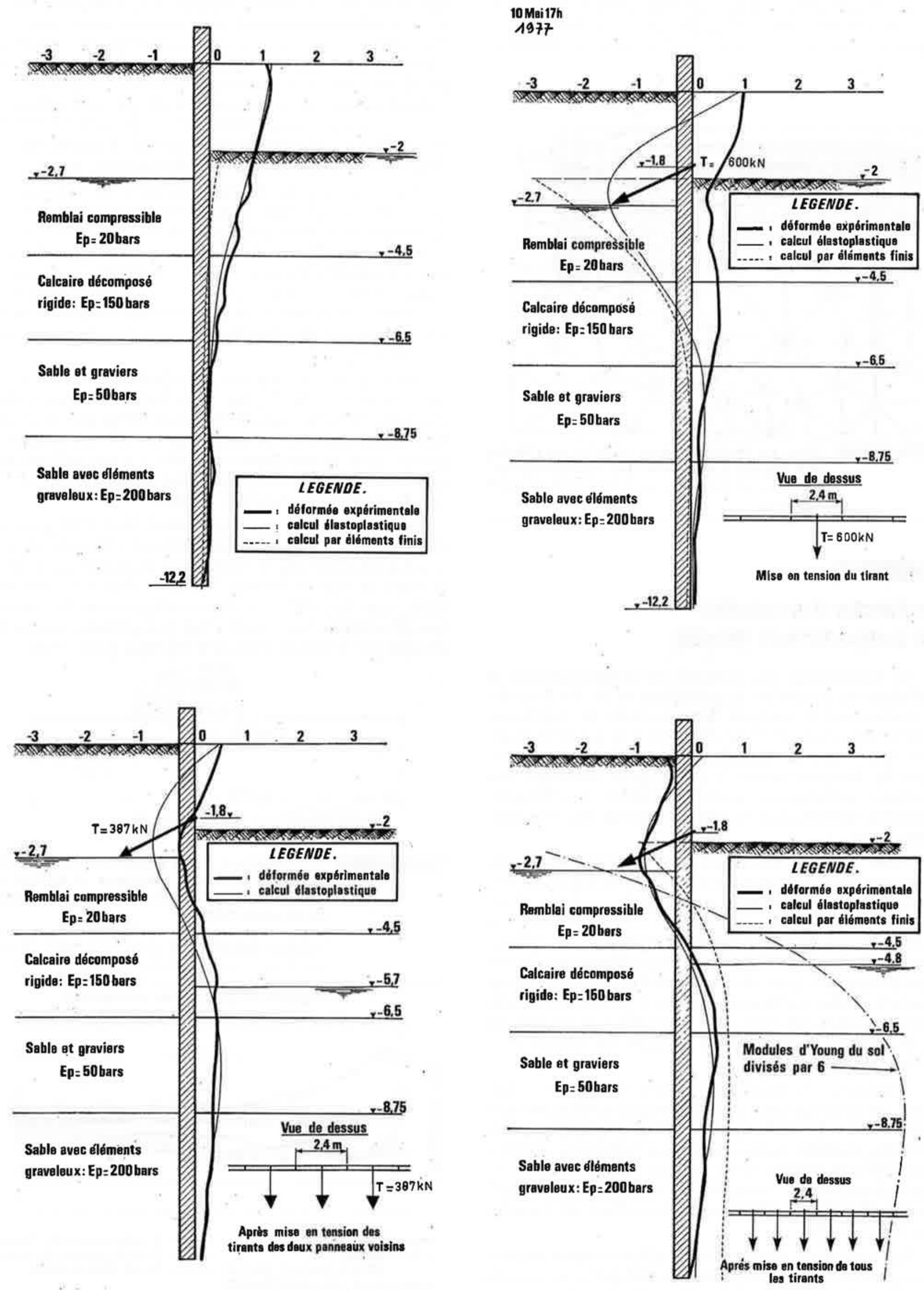

F16.23 Comparaison entre déformées calculées et mesurées du mur de quai de Deauville lors des premières phases d'excavation et de mise en tension des tirants $\left(1 \mathrm{bar}=10^{5} \mathrm{~Pa}\right)$.

Comparison between Deauville quay wall calculated and measured deflections during first excavation and tensioning stages. 
le cas de systèmes d'appuis présentant des contrastes de rigidités importants (Fig. 24) : de telles dispositions sont donc clairement à éviter en l'absence de justifications particulières.

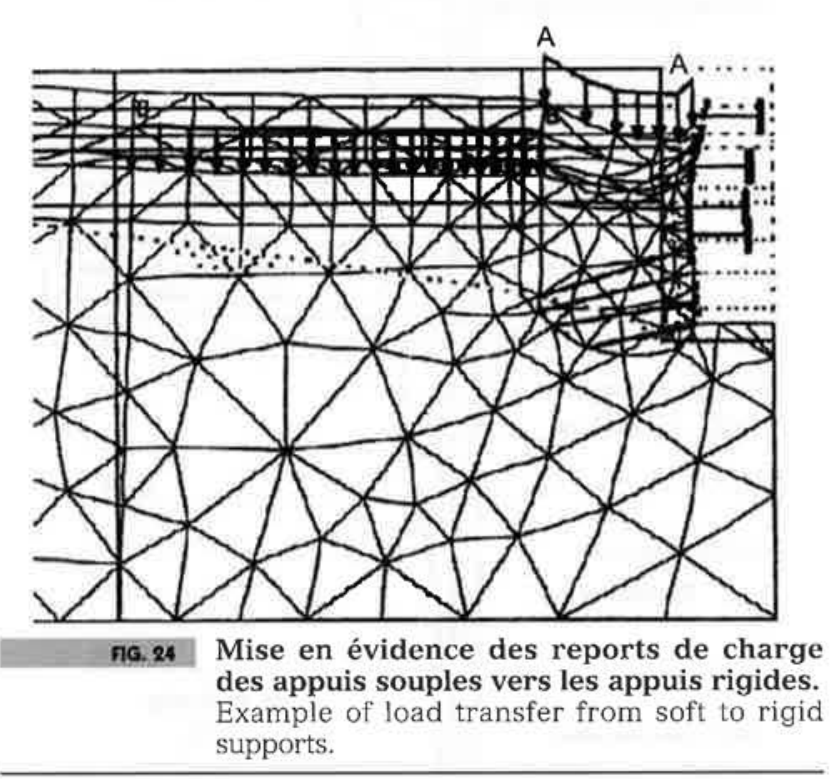

3.3

\section{Recherche d'un modèle de comportement général}

La multiplicité des modèles de comportement, la difficulté de connaître leurs limites et de les prendre correctement en compte dans le cadre de méthodes simplifiées amènent naturellement à se poser la question, qui n'est pas nouvelle, de la pertinence d'approches de dimensionnement empiriques alors que des modèles numériques sont disponibles, qui permettraient de prendre en compte la totalité des mécanismes en jeu.

Cette question est d'autant plus cruciale que les praticiens sont effectivement, qu'ils le veuillent ou non, ( condamnés ) à utiliser la méthode des éléments finis dans le cas de projets complexes, qui ne peuvent être correctement appréhendés à partir des seules méthodes traditionnelles : il peut dès lors s'avérer dangereux d'avoir recours, pour dimensionner des projets exceptionnels, à des méthodes qui n'ont pas été suffisamment validées, ou dont les paramètres n'ont pas été suffisamment calibrés dans le cadre de projets courants.

L'efficacité du modèle « poutre sur appuis élastiques ») pour les projets courants explique bien évidemment le peu d'efforts qui ont été déployés pour calibrer des modèles numériques plus généraux, exercice plus difficile compte tenu du plus grand nombre de paramètres en jeu, et surtout de la disponibilité insuffisante d'essais de laboratoire permettant de mesurer ces paramètres.

Le calibrage à partir du suivi géotechnique de projets courants n'a donc pu être tenté qu'au moyen de modèles simplifiés, dont les paramètres sont plus faciles à corréler avec l'essai pressiométrique, qui est toujours le plus pratiqué en France à l'heure actuelle.

On a ainsi facilement mis en évidence que, dans le cas d'un projet simple relevant du modèle classi- que « poutre sur appuis élastiques », l’ordre de grandeur du déplacement mesuré pouvait généralement être retrouvé avec une loi de comportement simplifiée reposant sur l'élasticité linéaire et le critère de rupture de Mohr-Coulomb, à condition d'utiliser une valeur du module d'Young relativement élevée par rapport à celles déduites des recommandations de Ménard (1965), qui définit $\mathrm{E}_{\mathrm{M}} / \alpha$. comme le « module d'Young du sol dans un champ de contraintes de compression ».

Il y a de ce point de vue parfaite concordance, ce qui n'est pas anormal, entre les calculs reposant sur la théorie de l'élasticité linéaire et ceux reposant sur la théorie du coefficient de réaction, qui n'est rien d'autre qu'une simplification de l'élasticité linéaire : les rétroanalyses associées au suivi géotechnique d'ouvrages courants conduisent ainsi généralement, dans les deux cas, à un ordre de grandeur du module d'Young supérieur à $4 . \mathrm{E}_{\mathrm{M}} / \alpha$.

On a ainsi pu montrer, en appliquant la formule empirique $\mathrm{k}=3,6 . \mathrm{E}_{\mathrm{M}} /(\alpha$. a) déduite de l'observation d'écrans de soutènement (Schmitt, 1995) au cas d'une semelle de fondation et en comparant le résultat obtenu à des calculs numériques conduits en élasticité linéaire, que le module d'Young équivalent était de l'ordre de 4,7. $\mathrm{E}_{\mathrm{M}} / \alpha$ (Fig. $25 ;$ Schmitt, 1998).

De même, un ordre de grandeur de 4 à $5 \mathrm{E}_{\mathrm{M}} / \alpha$ a pu être déduit de l'analyse par éléments finis de mesures du déplacement horizontal de quelques ouvrages, comme la Trémie Pasteur à Rouen (Fig. 30; Azoune, 2002 ; Escobar, 2001), la station d'épuration de Colombes (Plumelle et al., 2005), ainsi que certains ouvrages étudiés par d'autres auteurs (Phienwej et al., 1998).

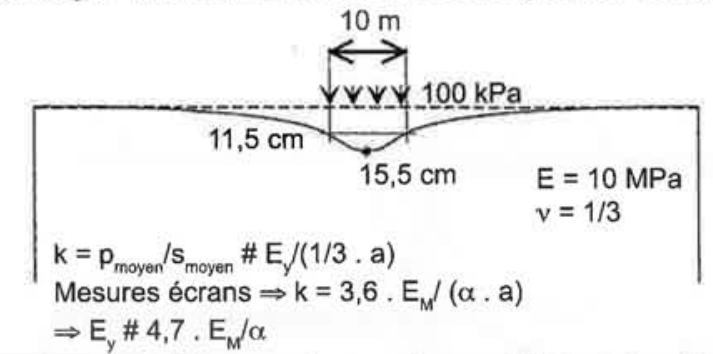

F16. 25 Comparaison entre coefficient de réaction déduit d'un calcul élastique et déduit de I'observation du déplacement des écrans de soutènement.

Comparison between coefficients of subgrade reaction derived from elastic calculation results and from measurements of walls displacemnts,

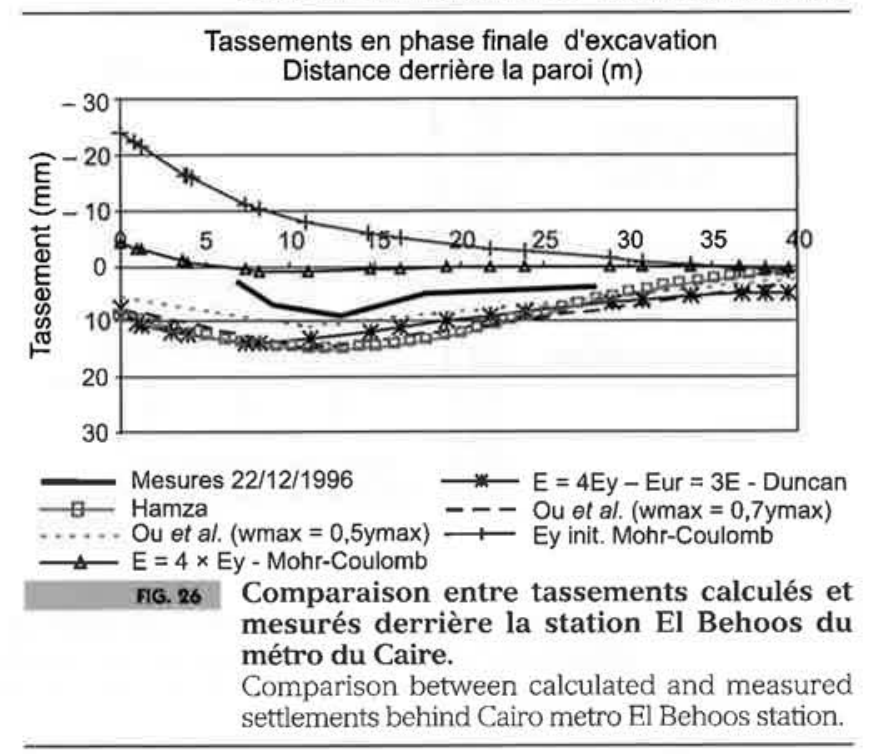




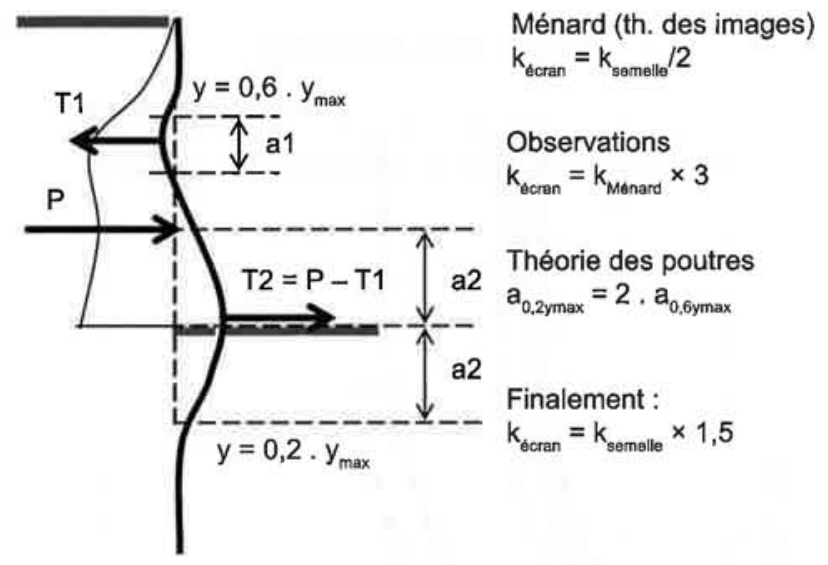

F6.27 Comparaison théorique entre les expressions du coefficient de réaction du sol à appliquer selon qu'il est mobilisé par un écran de soutènement ou par une semelle de fondation.

Theoretical comparison between relevant formulations for the coefficient of subgrade reaction considering it is mobilised either by a retaining structure or by a shallow foundation.

Ceci étant, l'utilisation de l'élasticité linéaire dans le cadre d'un calcul numérique de portée générale constitue une limitation importante, dans la mesure où seuls les déplacements horizontaux peuvent alors être exploités. En effet, l'impossibilité de distinguer le comportement du sol en chargement et en déchargement conduit à exagérer de façon irréaliste le soulèvement du sol provoqué par l'excavation, ainsi que cela a pu être mis en évidence par comparaison avec des mesures de tassements effectuées au voisinage de certaines des stations de la ligne 2 du métro du Caire (Fig. 26 ; Bazin et Schmitt, 2001)

De plus, cette loi simplifiée ne permet pas non plus de simuler à la fois le comportement d'un soutènement et celui d'une fondation, dans la mesure où la synthèse des suivis géotechniques pratiqués pour ces deux types d'ouvrages conduit à des modules d'Young équivalents généralement plus élevés (Fig. 27 ; Schmitt, 1998) dans le cas d'un soutènement (pour lequel, comme indiqué précédemment, on constate en général que $\mathrm{E}_{\mathrm{Y}}=4$ à 5 . $\mathrm{E}_{\mathrm{M}} / \alpha$ ) que dans le cas d'une fondation (pour laquelle on constate en général, si l'on exclut le cas des argiles molles pour lesquelles les valeurs peuvent être nettement plus faibles comme indiqué au \$3.2.1, que $E_{Y}=1$ à $4 . E_{M} / \alpha$; Combarieu, 2006).
Ainsi la figure 28 compare, pour différentes valeurs de la largeur a d'une semelle de fondation, le rapport entre le tassement obtenu par application de la théorie de l'élasticité linéaire, en supposant le module d'élasticité du sol égal au rapport $\mathrm{E}_{\mathrm{M}} / \alpha$ du module pressiométrique au coefficient de structure du sol, et celui obtenu par application directe des règles pressiométriques (Ménard, 1965).

L'accroissement important de ce rapport avec la largeur de la semelle traduit, selon Ménard, l'effet de la non linéarité du comportement du sol, qui le rend d'autant plus raide qu'il est plus éloigné, donc plus faiblement sollicité par la fondation.

Il n'en demeure pas moins que l'extrapolation, à une fondation souple de dimensions importantes, d'une formulation déduite du chargement de fondations rigides de moins de $2 \mathrm{~m}$ de largeur mériterait probablement quelques réserves, et ce d'autant plus que le passage à la limite consistant à appliquer les recommandations proposées par ailleurs par Ménard pour calculer le tassement sous le centre d'une fondation souple de grande largeur, à savoir un remblai reposant sur une couche de sol compressible, conduit à un rapport de tassements non plus supérieur, mais inférieur à 1 puisqu'il est alors proposé d'effectuer le calcul en assimilant à $\mathrm{E}_{\mathrm{M}} / \alpha$ le module de déformation vertical du sol, qui serait en l'occurrence non plus son module d'Young mais son module oedométrique (Gambin, 2005 ; Ménard, 1965).

Cette différence entre la raideur apparente du sol vis-à-vis des ouvrages de fondation et de soutènement remet d'ailleurs en cause l'explication qui avait initialement pu être proposée pour expliquer les fortes valeurs des coefficients de réaction déduites du suivi des ouvrages de soutènement comparativement aux ordres de grandeur antérieurement proposés par Terzaghi et Ménard, et qui reposait exclusivement sur la non linéarité du comportement du sol (Schmitt, 1984).

La non-linéarité est en effet une caractéristique intrinsèque du terrain, indépendante du type d'ouvrage géotechnique, et il n'y a aucune raison de penser que les ouvrages de fondation soient systématiquement associés à un taux de mobilisation du sol, en « butée verticale », plus élevé que les ouvrages de soutènement qui le sollicitent en « butée horizontale ».

En revanche, une différence fondamentale est que le sol, s'il est généralement sollicité de façon monotone par un ouvrage de fondation, se trouve systématique-

- Es Ménard $/\left(E_{M} / \alpha\right)$

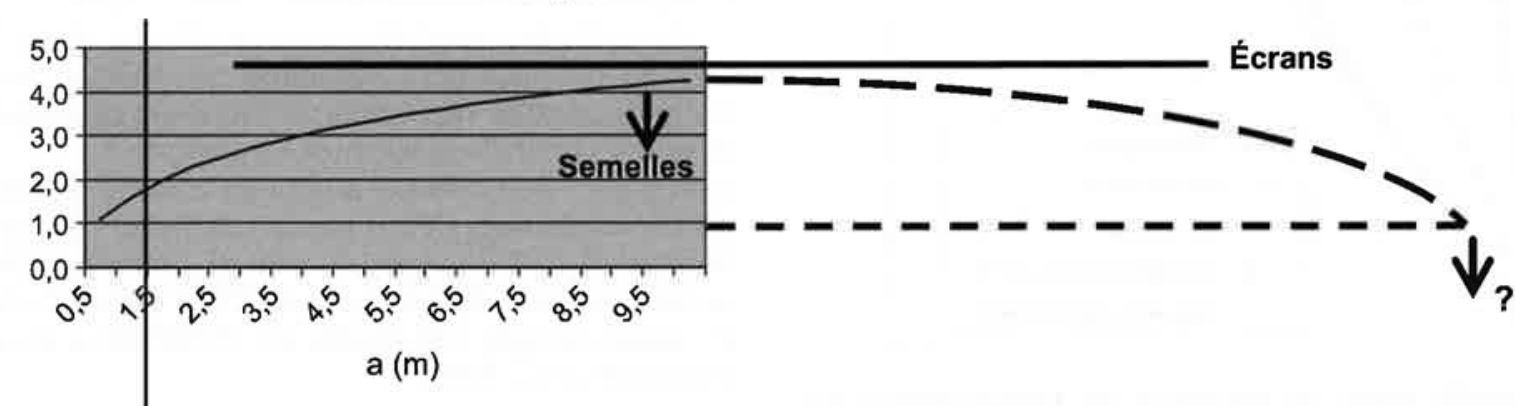

Domaine expérimental

Fc. 28 Synthèse rapide des retours d'expérience permettant de quantifier le rapport entre module équivalent du sol et module pressiométrique.

Short synthesis of site results relative to the actuel ratio between equivalent soil modulus and pressuremeter modulus. 
ment sollicité en déchargement (excavation), puis en rechargement (mise en butée) dans le cas d'un écran de soutènement, ce qui induit inévitablement un comportement plus raide ; l'usage de modèles numériques amène d'ailleurs à constater que, dans le cas d'une loi de comportement plus complexe que l'élasticité linéaire, l'incidence des paramètres de déchargement sur le déplacement calculé d'un écran $\left(E_{u r^{\prime}} v_{u r^{\prime}} \kappa \ldots\right)$ est au moins aussi grande, sinon plus, que celle des paramètres de premier chargement $(E, v, \lambda \ldots)$.

Les calculs effectués pour rendre compte du comportement observé de la Trémie Pasteur, à Rouen, ont ainsi mis en évidence, toutes choses égales par ailleurs, que l'hypothèse d'un comportement linéaire ou hyperbolique en premier chargement était sans incidence sensible, tandis que le seul fait de multiplier par 3 le module d'élasticité en déchargement-rechargement $E_{u r}$ suffisait à diviser le déplacement par 2 (Fig. 29 ; Escobar, 2001).

Ce constat renforce d'ailleurs la pertinence de la théorie du coefficient de réaction, qui caricature certes l'élasticité linéaire, mais en se limitant à un domaine de déchargement-rechargement qui se trouve être, précisément, celui dans lequel le comportement du sol peut être valablement approché par un tel modèle.

Par contre, le coefficient de réaction a l'immense mérite de ne pas prétendre représenter le comportement du sol vis-à-vis d'un mode de sollicitation bidirectionnel, contrairement au cas d'un calcul aux éléments finis conduit en élasticité linéaire, et qui serait censé représenter avec un jeu unique de paramètres le comportement du sol aussi bien vis-à-vis d'une fondation que d'une excavation.

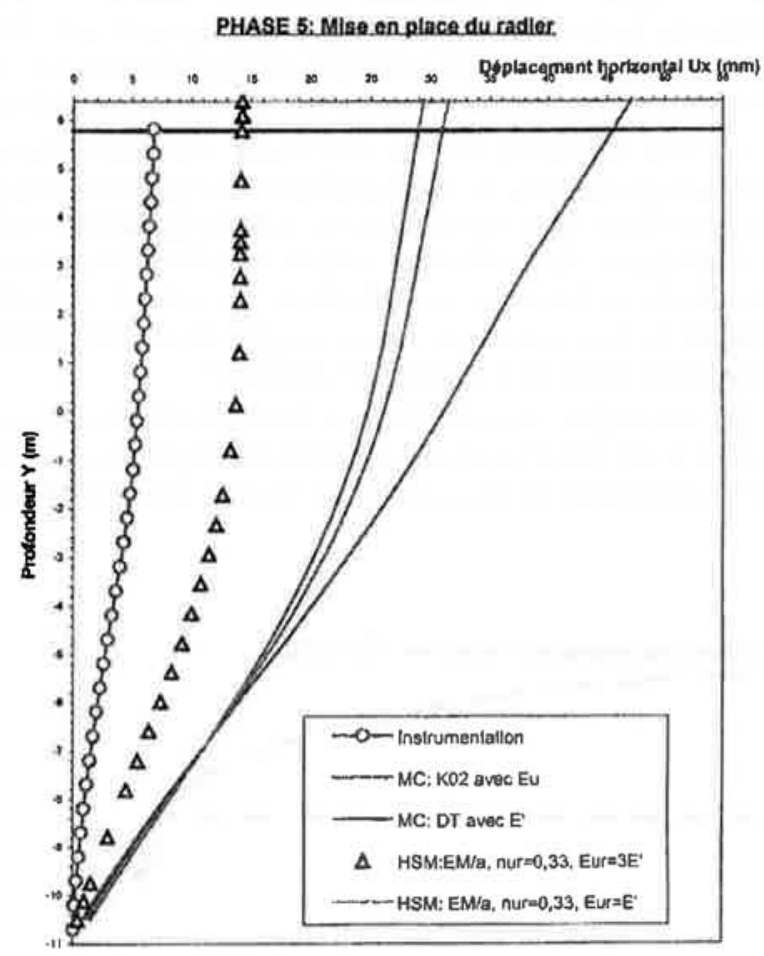

10.29 Mise en évidence de l'importance du module de déchargement-rechargement du sol comparativement au modèle retenu pour le premier chargement du sol. Importance of the choice of the unloadingreloading modulus compared with that of the primary loading model.

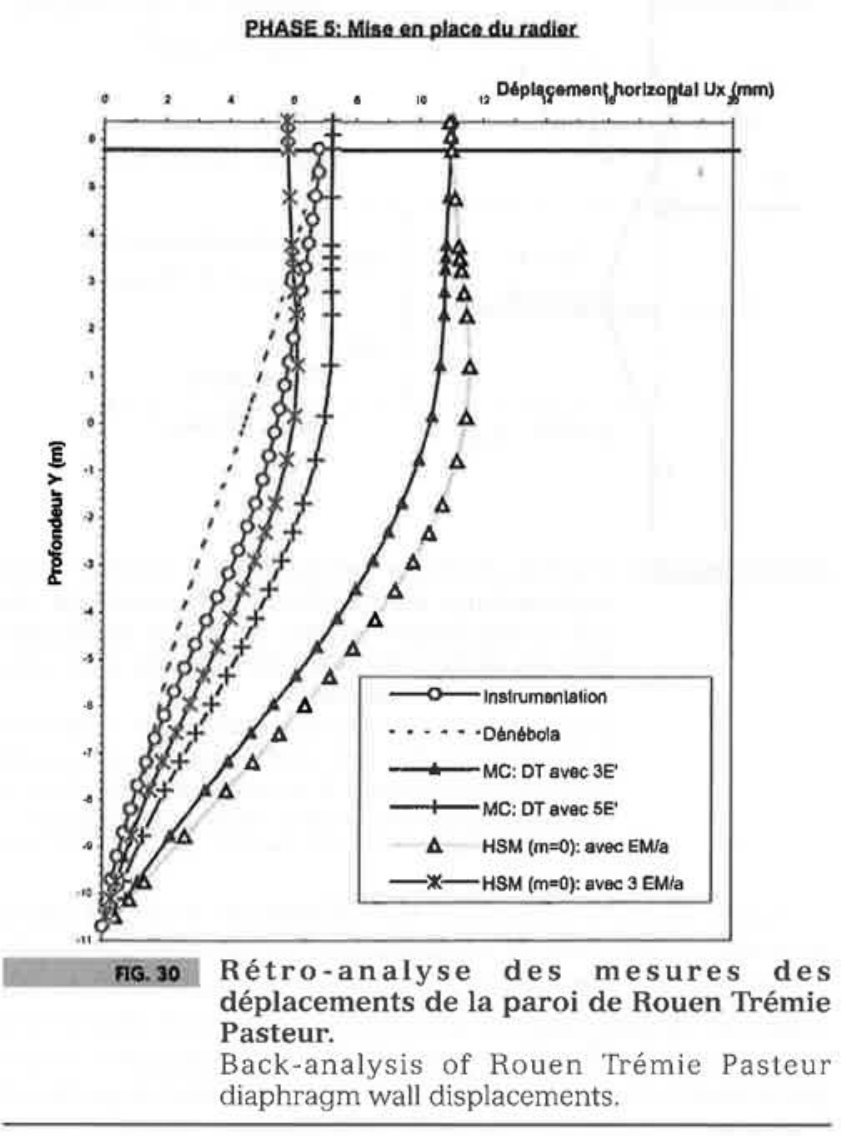

Ceci justifie que les calibrages des modèles numériques dans le domaine des écrans de soutènement soient maintenant le plus souvent effectués vis-à-vis de modèles non linéaires (Cam-Clay, loi hyperbolique...).

Dans le cadre du modèle hyperbolique, les calages récemment effectués comparativement aux mesures de déformations des parois moulées de la trémie Pasteur, à Rouen (Fig. 30 ; Azoune, 2002 ; Escobar, 2001), et de Colombes (Plumelle et al., 2005 ; Serrai, 2001), ont permis de mettre en évidence que le module $\mathrm{E}_{50^{\prime}}$ qui représente théoriquement le module de déformation sécant à $50 \%$ de la rupture, et est généralement associé, à défaut de mesures dignes de ce nom, à un module de déchargement-rechargement $\mathrm{E}_{\mathrm{ur}}$ trois fois plus élevé, se trouve être d'un ordre de grandeur 2 fois plus faible que le module d'Young qui permettrait de retrouver le même déplacement dans le cadre d'un calcul en élasticité linéaire, soit $\mathrm{E}_{50}$ de l'ordre de 2 à 3. $\mathrm{E}_{\mathrm{M}} / \alpha$ comparativement à $\mathrm{E}_{\mathrm{Y}}=4$ à $5 \cdot \mathrm{E}_{\mathrm{M}} / \alpha$.

Il est intéressant de remarquer que, dans le cas d'un sol normalement consolidé, pour lequel le coefficient de poussée des terres au repos est classiquement estimé à partir de la formule empirique $K_{0}=1-\sin \varphi$, tandis que l'état critique atteint en conditions triaxiales est classiquement décrit par le coefficient de butée de Rankine $\mathrm{K}_{\mathrm{p}}=(1+\sin \varphi) /(1-\sin \varphi)$, on peut facilement montrer que le déviateur dans l'état initial du terrain en place est égal à la moitié du déviateur à la rupture (Plumelle et al., 2005).

On peut donc en première approximation admettre que le module à prendre en compte vis-à-vis d'un chargement vertical monotone (cas d'un projet de fondation) serait le module tangent à $50 \%$ de la rupture, soit $\mathrm{E}_{\text {50tangent }}=\mathrm{E}_{\text {sosécant }} / 2$ dans l'hypothèse d'un comportement hyperbolique (Fig. 31). 


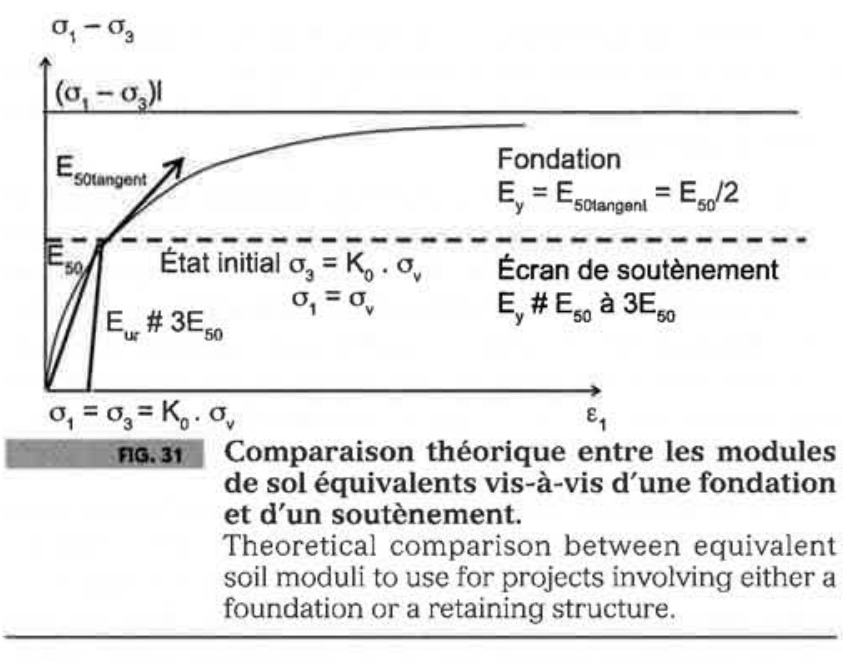

Dans la mesure où le comportement du sol sollicité en décompression/recompression (excavation/ mise en butée) par un écran de soutènement est en partie décrit par un module de déchargement/rechargement $E_{u r}=3 . E_{50 \text { sécant' }}$ on peut aisément expliquer un comportement notablement plus raide que vis-à-vis d'une fondation : il n'est finalement pas illogique qu'un calcul utilisant un modèle hyperbolique avec $E_{50}$ de l'ordre de $2 . E_{M} / \alpha$, associé à $E_{u r}=6 . E_{M} / \alpha$, conduise à des résultats du même ordre de grandeur qu'un calcul mené en élasticité linéaire avec $E_{Y}=4 . E_{M} / \alpha$, sachant que le même modèle hyperbolique conduirait, vis-à-vis du chargement vertical induit par une fondation, à un résultat équivalent à $\mathrm{E}_{\mathrm{Y}}=\mathrm{E}_{50} / 2=\mathrm{E}_{\mathrm{M}} / \alpha$.

Les valeurs, plus élevées que $\mathrm{E}_{\mathrm{M}} / \alpha$, observées dans le cas de semelles rigides de faible largeur pourraient alors être expliquées par l'anisotropie de constitution du sol, toujours ignorée dans les modèles de calcul, probablement à tort (Combarieu, 2006 ; Ménard, 1965).

Ces ordres de grandeur, qui ne concernent en toute rigueur que des terrains normalement consolidés, doivent être toutefois considérés avec la plus extrême prudence en raison du nombre limité d'observations sur lesquelles ils reposent, d'une part, et, d'autre part, de l'incertitude inhérente aux calages réalisés a posteriori, compte tenu du nombre important de paramètres en jeu et du fait que ces paramètres ne sont généralement pas mesurés.

Dans le cas de la paroi de Colombes, d'importants efforts de rétro-analyse ont été accomplis pour essayer de retrouver, en utilisant la méthode des éléments finis, la même superposition avec les déformées mesurées par inclinométrie que celle obtenue quelques années auparavant en utilisant de simples calculs aux coefficients de réaction (Fig. 32 ; Plumelle et al., 2005 ; Serrai, 2001 ; Londez et al., 1997).

Force est de constater que, si l'ordre de grandeur des déplacements a parfaitement pu être retrouvé en utilisant les ordres de grandeur des modules de déformation indiqués précédemment $\left(\mathrm{E}_{\mathrm{Y}}=4 . \mathrm{E}_{\mathrm{M}} / \alpha\right.$ dans le cas d'un modèle avec comportement élastique et linéaire, $E_{50}=2 . E_{M} / \alpha$ dans le cas d'un modèle hyperbolique), la superposition des déformées proprement dites n'a jamais pu être obtenue dans l'ensemble des phases de réalisation en utilisant un jeu de paramètres unique.

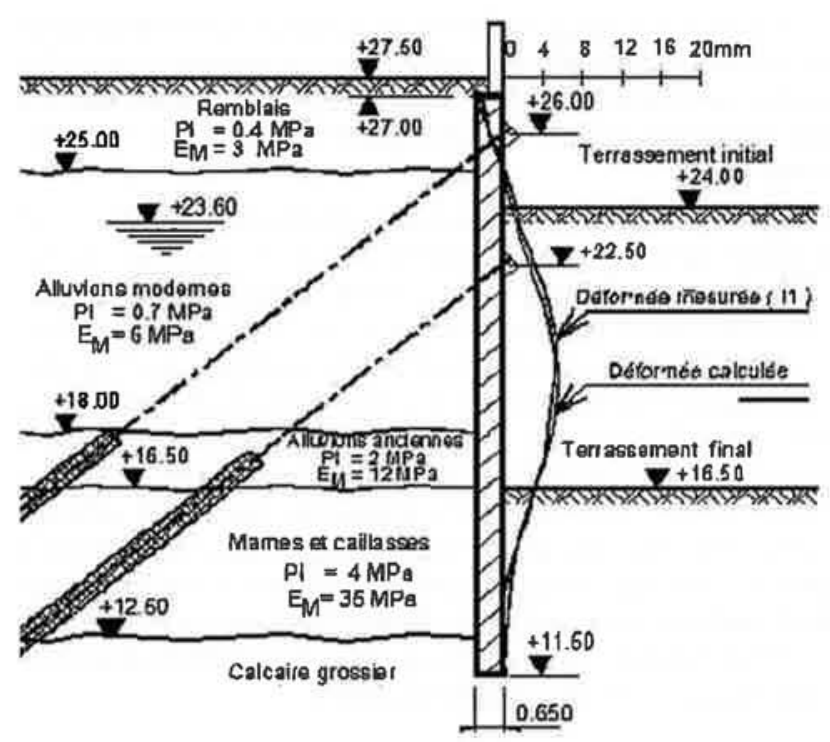

F6. 32 Comparaison entre les déplacements prévus et mesurés de la paroi moulée de Colombes, en utilisant des calculs aux coefficients de réaction.

Comparison between predicted and measured displacements of Colombes diaphragm wall using coefficients of subgrade reaction.

En particulier, la rentrée du soutènement dans le terrain en tête lors des phases de terrassement profond, pour lesquelles l'appui des tirants agit comme un centre de rotation, ce qui est nettement mis en évidence par les mesures de déplacement et les calculs aux coefficients de réaction, n'a jamais pu être retrouvé par le calcul aux éléments finis (Fig. 33), sauf à réduire les paramètres de déformabilité des terrains de tête, au prix de déplacement cette fois incorrects lors des premières phases de terrassement et de mise en tension.

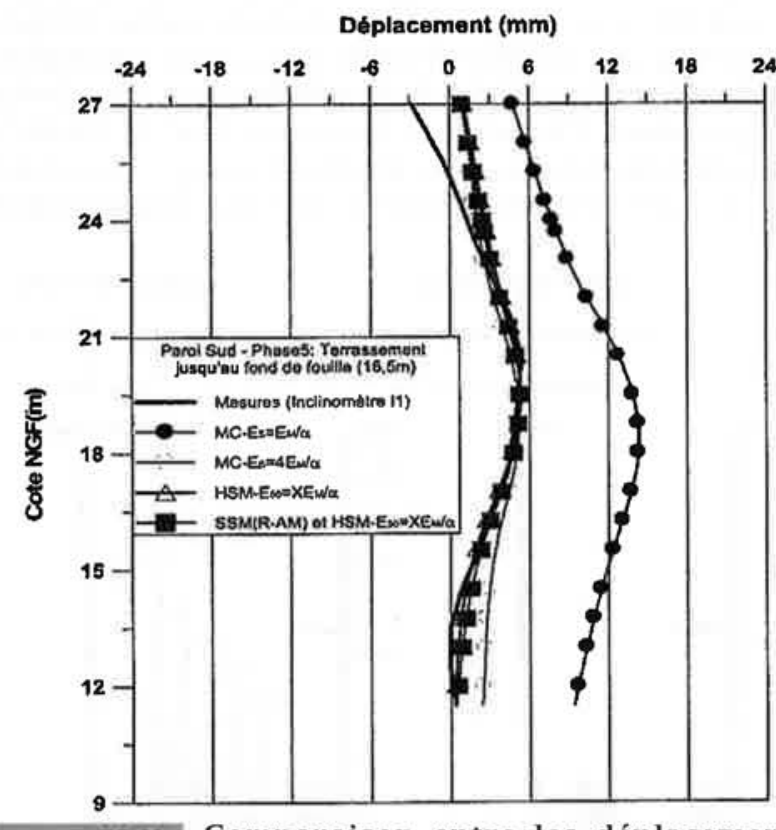

ทต. 33 Comparaison entre les déplacements prévus et mesurés de la paroi moulée de Colombes, en utilisant la méthode des éléments finis.

Comparison between predicted and measured displacements of Colombes diaphragm wall using finite elements method. 
Ce résultat pour le point paradoxal (on s'attendrait au contraire à ce qu'une méthode numérique approche mieux la réalité que des calculs réputés simplistes) n'a pas encore à ce jour pu être expliqué de façon satisfaisante.

Ces exemples isolés, même significatifs, demandent évidemment à être complétés par de nombreux autres calages, ce qui démontre une nouvelle fois l'importance du suivi géotechnique pour progresser efficacement dans la connaissance du comportement des ouvrages et en permettre une modélisation réaliste. La nécessité de parvenir à un calage précis non seulement vis-à-vis des déplacements, mais encore des déformations, résulte non pas d'un soucis de précision scientifique, mais du fait que les calculs servent également à déterminer les sollicitations, notamment les moments fléchissants qui sont directement proportionnels à la courbure des écrans, et vis-à-vis desquels ces derniers vont finalement être dimensionnés.

\section{4}

\section{Suivi de la réalisation des ouvrages}

Les exemples précédents ont démontré la nécessité du suivi géotechnique pour mieux connaître les modèles de comportement, ainsi que leurs limites, et caler les modèles de calcul et paramètres associés.

Ils permettent également parfois de mettre en évidence des phénomènes que les calculs ignorent systématiquement, à savoir l'effet de la réalisation même des ouvrages géotechniques : mouvements associés aux vibrations provoquées par le battage, à l'excavation des parois moulées, à la perforation des pieux et tirants, etc.

D'autres phénomènes, également impossibles à quantifier actuellement, peuvent également être mis en évidence. Ainsi, le comportement de la paroi moulée de la station El Behoos, précédemment citée, a également été suivi pendant l'injection du radier d'étanchéité (Fig. 34). L'(c effet de paroi ) décrit par Cambefort (1967), à savoir le déplacement de la paroi sous l'effet des pressions d'injection et les remontées de coulis le long de l'interface, a ainsi pu être constaté, aussi bien par le suivi des déformations que par l'observation
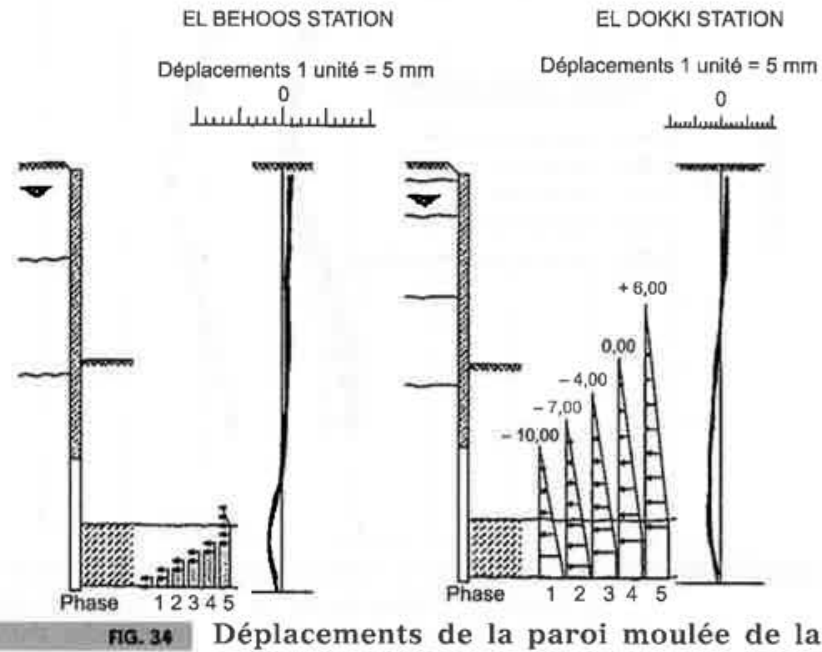

Déplacements de la paroi moulée de la station El Behoos du métro du Caire pendant l'injection du radier.

Displacements of El Behoos station diaphragm wall while grouting at slab level. ultérieure du parement au moment des terrassements : cet effet a été modélisé a posteriori, mais on ne dispose encore à l'heure actuelle d'aucune méthode véritablement ( prédictive ».

Les capteurs de contraintes totales disposés le long de la paroi moulée de la trémie Pasteur, à Rouen (Ducas, 2001), ont permis de confirmer la compensation de la diminution des contraintes horizontales pendant l'excavation de la paroi moulée par l'augmentation, sensiblement supérieure, des contraintes provoquées par le bétonnage, ainsi que cela avait déjà été établi au port du Havre (Reynaud et Rivière, 1981).

Il a également été montré qu'il fallait quelques mois avant que l'augmentation de contraintes associée au bétonnage ne se relaxe suffisamment pour que l'on retrouve approximativement la pression initiale des terres au repos, ce qui met en évidence un autre paramètre susceptible d'avoir une influence sur les sollicitations appliquées à l'ouvrage, à savoir le délai entre la réalisation de la paroi moulée et le début des terrassements.

Ce sont de telles mesures qui permettent de faire petit à petit progresser les méthodes de dimensionnement des ouvrages géotechniques, mais qui permettent aussi de comprendre que ces méthodes ne peuvent (et ne pourront peut-être jamais) être considérées comme suffisantes, d'où il résulte que le suivi géotechnique, au-delà de son rôle prédictif, doit également être considéré comme une partie intégrante du processus de conception et de réalisation.

\section{4 \\ Maîtrise de la sécurité des ouvrages géotechniques}

\section{1}

\section{Respect des états limites de service}

Comme on l'a vu, la nécessité du suivi géotechnique résulte en grande partie de l'imprécision avec laquelle les calculs permettent de prévoir le comportement réel des ouvrages géotechniques.

Ceci étant, l'intensité de ce suivi doit bien évidemment être modulée en fonction de plusieurs critères :

- l'incertitude, généralement fonction de la complexité du projet et du site ;

- les conséquences de cette incertitude, en termes de risques non seulement vis-à-vis du fonctionnement des ouvrages (états limites de service), mais encore vis-àvis de leur stabilité (états limites géotechniques) et de leur résistance (états limites structurels).

A cet égard, on notera que l'Eurocode 7 distingue effectivement le (c suivi géotechnique ), applicable au cas des ouvrages courants à classe de conséquence limitée, et la « méthode observationnelle », plus justement appelée « dimensionnement interactif », dans laquelle les mesures ne servent plus seulement à s'assurer du bon fonctionnement des ouvrages et affiner ultérieurement les modèles de calcul, mais vont également permettre d'orienter les travaux en fonction de critères prédéfinis (Allagnat, 2005 ; AFNOR, 2005 ; Schlosser et Schmitt, 2007). 
Cette stratégie plus contraignante va être mise en œuvre dans des circonstances bien spécifiques, qui devront être détectées suffisamment en amont du processus de réalisation pour que les dispositions qui s'imposent puissent être anticipées par l'ensemble des intervenants, en termes de moyens de calcul, de mesure, d'interprétation, et finalement d'adaptation du projet, comme cela est clairement décrit dans le guide français (Allagnat, 2005).

La mise en œuvre de cette stratégie dépend en pratique des raisons qui ont conduit à recourir à une procédure de dimensionnement interactif (Schlosser et Schmitt, 2007). En effet, lorsqu'il s'agit non pas de faire face à un risque technique important mais de répondre à un souci d'optimisation du projet, il est en général suffisant de procéder à des calculs supplémentaires tenant compte de différents jeux de paramètres, dont l'un réputé ( réaliste ») va permettre d'établir le projet de référence, tandis que l'autre réputé « pessimiste » va permettre de proposer un projet de confortement, que l'on mettra ou non en œuvre selon les résultats des mesures.

Dans le cas du projet de Nile City au Caire (Fig. 35 ; Schlosser et Schmitt, 2007), où le dimensionnement initial reposait sur la prise en compte des résultats de calculs aux éléments finis dans lesquels la pression d'eau sur l'écran était notablement réduite par l'effet du rabattement extérieur, le projet était grandement conditionné par la valeur du coefficient d'anisotropie de la perméabilité du sol.

La prise en compte d'un calcul plus optimiste que les approches traditionnelles, qui consistent à négliger prudemment l'effet incertain du rabattement extérieur, comme le recommande notamment la Norme Française relative au calcul des écrans de soutènement (AFNOR, 2009), n'a été admise que moyennant la mise en œuvre d'une procédure de dimensionnement interactif, dans laquelle la solution de confortement consistait à doubler le nombre de tirants initialement prévu au moyen de réservations supplémentaires insérées
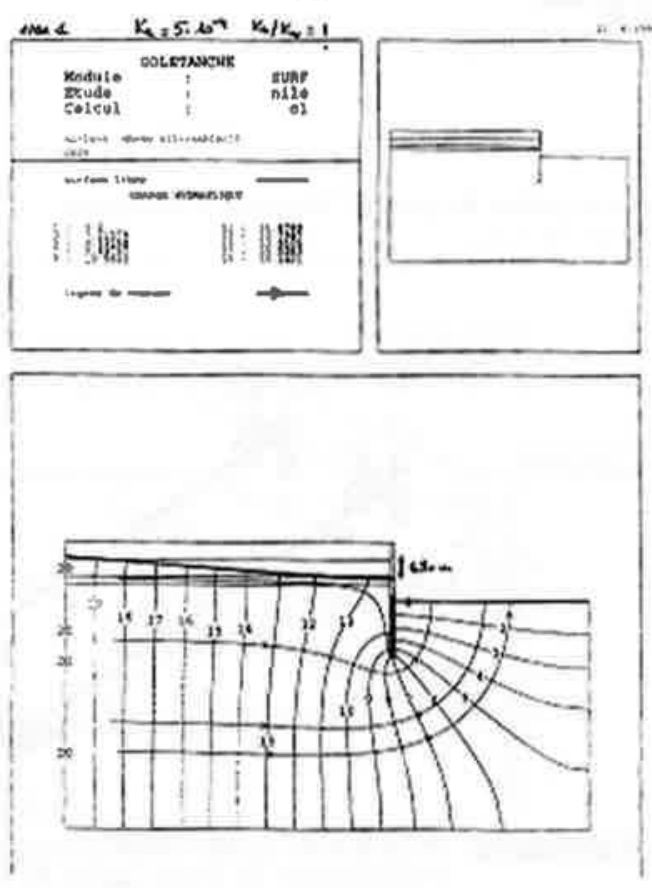

dans les cages d'armature. Le suivi effectué n'a pas permis en l'occurrence de réaliser l'économie escomptée, mais aura au contraire mis en évidence les dangers d'une utilisation insouciante de modèles numériques insuffisamment étalonnés.

Les choses sont plus complexes lorsque le souci premier est, non pas de rechercher une économie potentielle, mais de faire face à un niveau d'incertitude suffisamment élevé pour constituer un facteur de risque important : il s'agit alors, comme le prescrit l'Eurocode 7, de définir les limites du comportement acceptable de l'ouvrage, par référence non pas au calcul mais par exemple à la structure des ouvrages existants, si c'est le maintien de leur intégrité qui conditionne la définition des critères de déformation, et de comparer ces limites à celles du comportement prévisible, estimées si possible non pas par le calcul, réputé incertain donc par définition incapable de prédire lui-même ses propres limites, mais à partir d'expériences comparables résultant de suivis antérieurs.

La décision de recourir ou non à la méthode du dimensionnement interactif est souvent difficile, et peut même parfois n'être prise qu'en cours de travaux, sur la base des résultats de ce qui n'était au départ qu'un suivi géotechnique de routine, mais a finalement révélé des incertitudes plus importantes que prévu, ce qui démontre la nécessité de ne pas confondre suivi géotechnique et mesures sans suivi, ce qui est malheureusement trop fréquent mais ne présente en pratique aucun intérêt.

Il est important de remarquer que ce sont le plus souvent les déplacements et les déformations qui sont mesurés, éventuellement mais plus rarement les réactions d'appui (par exemple lorsque ceux-ci sont constitués par des tirants précontraints), ce qui se prête essentiellement à la détection et à la prévention d'états limites de service.

Un exemple intéressant est celui du projet « Le Minerve ») à Monaco (Fig. 36 ; Lavisse et al., 2007 ; Schlosser et Schmitt, 2007). Lors de sa réalisation, les

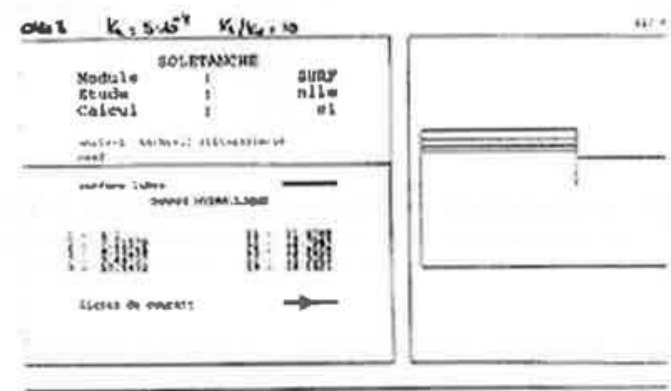

FG.35 Influence de l'anisotropie de perméabilité sur le rabattement extérieur à la paroi moulée du projet de Nile City.

Effect of hydraulic anisotropy on dewatering level behind Nile City project diaphragm wall. 


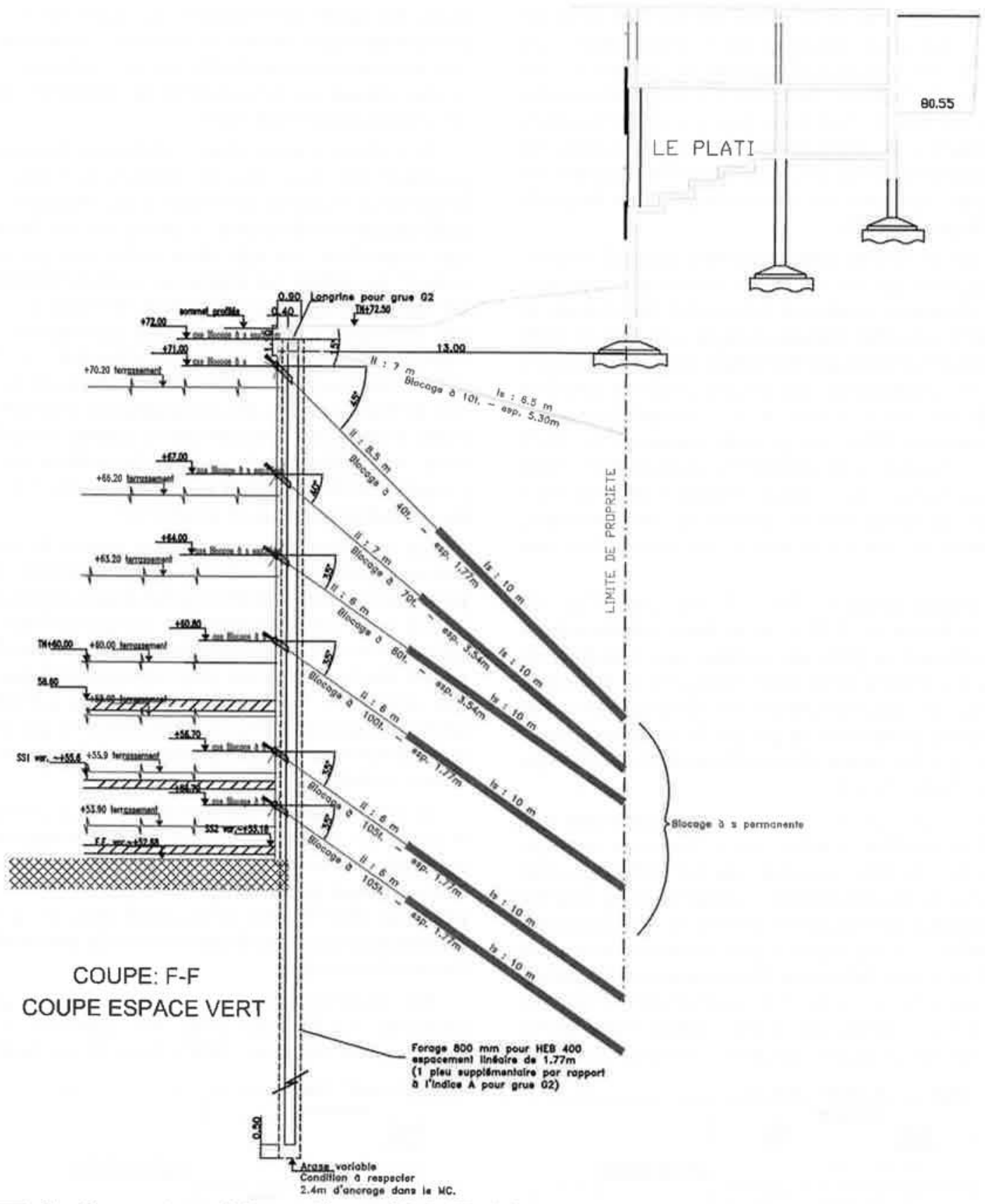

Fic.36 Soutènement ancré devant l'immeuble le Plati dans le cadre du projet Minerve à Monaco. Anchored wall in front of Le Plati building, Minerve project, Monaco.

déplacements, devant l'immeuble « Le Plati » réputé sensible, mais devant lequel les tirants étaient pour des raisons administratives limités à une longueur de $13 \mathrm{~m}$, ont pu être maîtrisés par l'augmentation du nombre de tirants précontraints initialement prévus dans la zone adjacente, non sujette à cette limitation de longueur, et ainsi par la création d'un point dur artificiel constituant le support d'un arc de décharge horizontal devant le Plati (Fig. 38), ce qui aura permis d'éviter la mise en œuvre dans cette zone de la solution de butonnage extrêmement contraignante qui avait été envisagée dans le cadre de la procédure de dimensionnement interactif (Fig. 37).

La figure 39 donne également l'exemple de la stratégie adoptée pour définir le seuil d'alerte, dans le but d'anticiper le seuil d'intervention lui-même, et ce dans chacune des étapes de réalisation de façon à prévenir le risque de découverte tardive, c'est-à-dire en phase

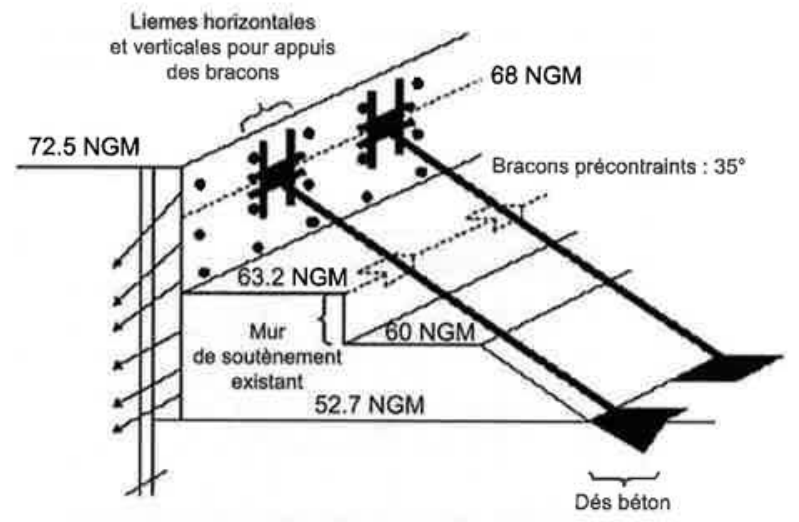

ต6. 37 Solution de confortement envisagée devant l'immeuble le Plati dans le cadre de la méthode observationnelle.

Reinforced project foreseen in front of Le Plati building, using the observational method. 


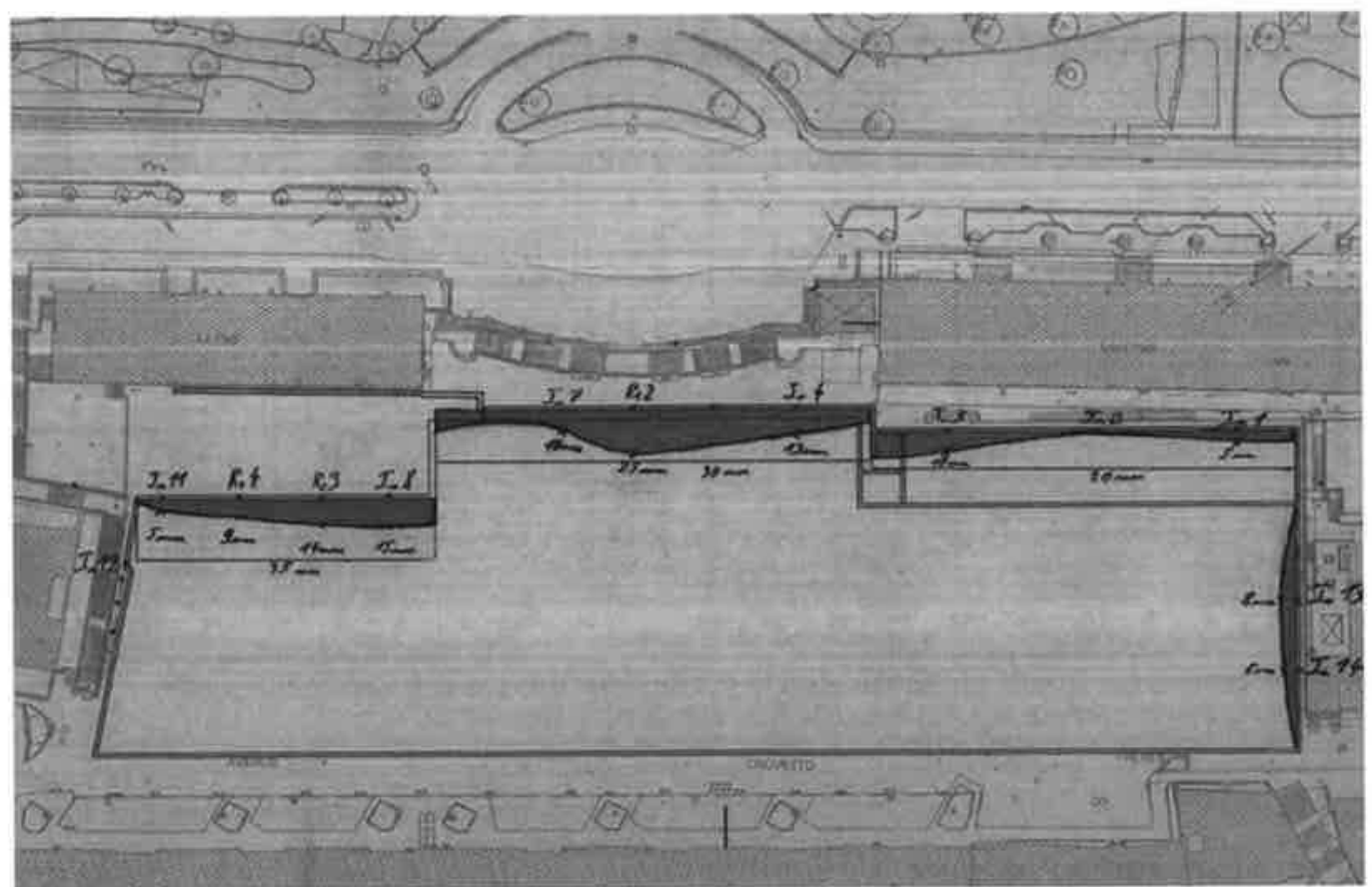

Déplacements mesurés pendant l'excavation nécessaire à la réalisation de l'immeuble le Minerve à Monaco.

Measured displacements while excavation during Le Minerve building works in Monaco.

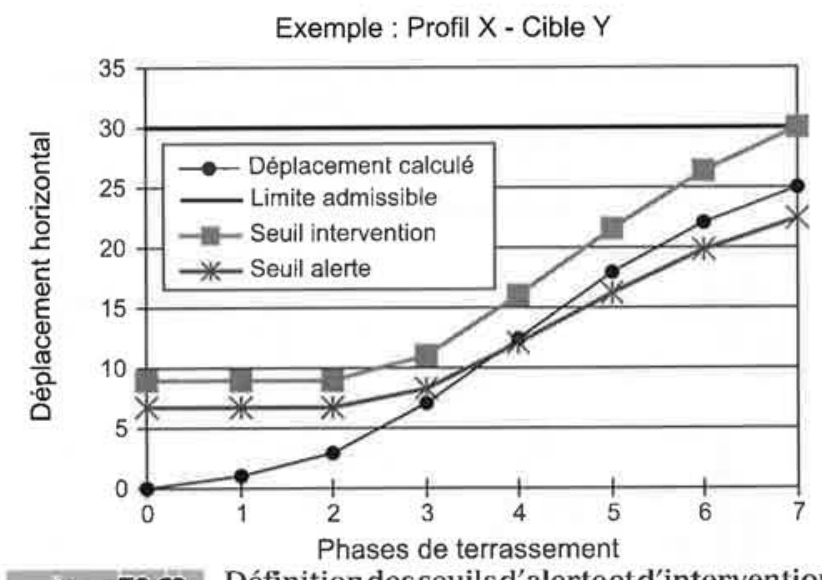

ต๑. 39 Définition desseuils d'alerteetd'intervention dans le cadre du projet Minerve.

Fixed displacements limits for Minerve project.

finale d'excavation seulement, du dépassement d'un seuil. On constate ainsi, pour ce projet sensible, que la courbe de définition des seuils d'alerte recoupe celle des déplacements calculés, ce qui a le mérite de placer d'emblée l'ensemble des intervenants en situation d'alerte permanente.

Pour revenir sur le problème crucial de l'évaluation du comportement prévisible à partir d'expériences comparables, comme prescrit par l'Eurocode 7 , le suivi géotechnique permet aussi de mettre en évidence l'évolution remarquable des techniques de réalisation des soutènements, depuis les résultats publiés par Peck et repris par Holtz et al. (1985;

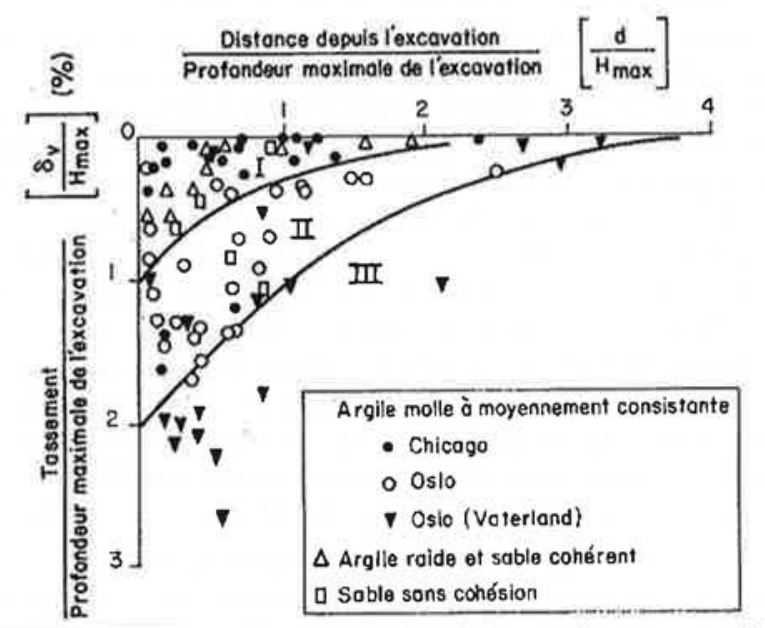

Tassements au voisinage des excavations en fonction de la distance au bord et de la profondeur maximale (d'après Peck, 1969). Settlements around excavations as a function of horizontal distance and excavation depth (according to Peck, 1969).

Fig. 40), faisant état de déplacements généralement de l'ordre de quelques pourcents de la hauteur terrassée, jusqu'à l'époque actuelle où le recours à des parois moulées de forte inertie et à des dispositifs d'appuis rigides ou précontraints permet d'obtenir de plus en plus souvent des déplacements limités à quelque $0,1 \%$ de la hauteur (Fig. 41 ; Marten, 2005).

Il est important de noter que des valeurs inférieures ou égales à $0,1 \%$ correspondent toujours à des terrains rocheux, ou à la mise en œuvre de parois de très forte inertie (à contreforts ou circulaires), ne relevant plus du domaine des écrans plans traditionnels. 

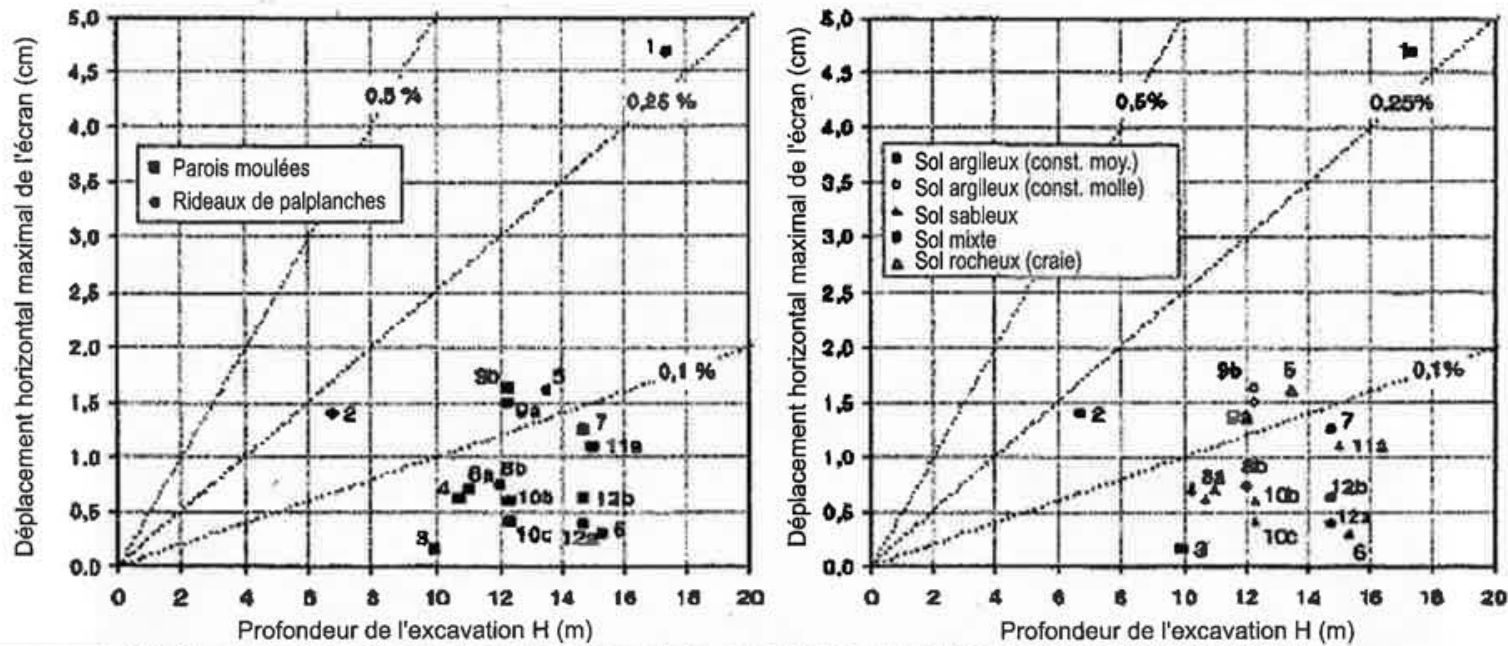

56.41 Déplacement horizontal en fonction de la profondeur de l'excavation (LCPC, 2005). Horizontal displacement as a function of excavation depth (LCPC, 2005).

\section{2}

\section{Prévention des états limites ultimes}

On admet bien souvent que la prévention d'un état limite de service est suffisante pour éviter l'occurrence d'un état limite ultime, ce qui n'est pourtant pas nécessairement le cas si les états limites en question ne relèvent pas exactement du même mécanisme.

Une question difficile est alors celle de la limite du comportement acceptable (dont l'Eurocode 7 exige la définition préalable) lorsque le recours au dimensionnement interactif a pour objectif d'éviter non plus l'occurrence d'un état limite de service, généralement exprimé comme on l'a vu en termes de déplacement fonction de la nature des structures avoisinantes, mais uniquement d'un état limite ultime, dont l'éloignement est réglementairement fixé par l'introduction dans le calcul de facteurs partiels appliqués aux actions et résistances, lesquels facteurs partiels échappent généralement à toute mesure directe, même s'il existe à l'évidence une certaine corrélation entre coefficients de sécurité et déplacements (Fig. 42 ; Holtz et al., 1985).

Le seuil d'alerte, sinon d'intervention, devrait alors être logiquement fixé par l'observation d'un comportement conforme aux prévisions, dans la mesure où les paramètres de calcul utilisés pour la justification du projet vis-à-vis d'un ELU sont généralement les mêmes que ceux utilisés pour l'étude du comportement prévisible.

Ceci peut sembler paradoxal dans la mesure où un comportement conforme aux prévisions témoignerait d'une remarquable maîtrise du calcul géotechnique, et constituerait donc au contraire un facteur rassurant. Il n'en demeure pas moins que le dépassement des limites du comportement prévu peut être considéré comme le début d'une incursion dans le domaine dangereux où l'on commence à ( consommer » les facteurs partiels, destinés à servir de ( tampon » entre l'état limite et la réalité.

Ce problème des limites du comportement acceptable vis-à-vis d'un ELU n'est actuellement traité par aucun texte, ce qui témoigne d'un certain embarras et du souci d'éviter une recommandation drastique mais quelque peu absurde qui consisterait à faire coïncider comportement prévisible et comportement acceptable, et imposer un renforcement du projet dès lors que le comportement mesuré s'avère conforme aux prévisions!

La pratique reconnaît bien implicitement, mais sans pouvoir l'écrire et encore moins le quantifier, un certain droit à la consommation des facteurs partiels, qui servent toujours dans une certaine mesure à compenser un peu l'incertitude inhérente au calcul géotechnique, à condition de savoir rester dans les limites du ( raisonnable )...

Ce qui semble en pratique raisonnable serait de considérer les limites du comportement prévu non pas comme seuil d'intervention, mais comme « seuil de

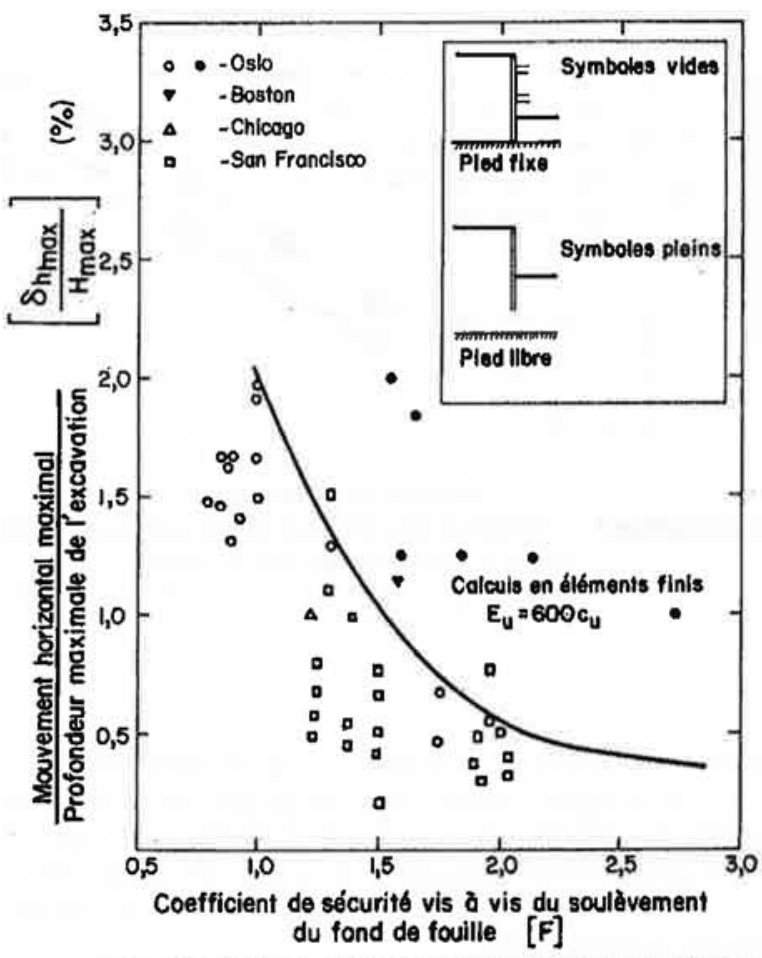

16. 42 Relation entre le déplacement latéral du soutènement et le coefficient de sécurité vis-à-vis du soulèvement du fond de l'excavation dans le cas des argiles (d'après Clough et Schmidt, in Holtz et al., 1985).

Horizontal displacement as a function of safety factor relative to excavation bottom heave in clay (according to Clough and Schmidt). 
vigilance m) à partir duquel les différences entre comportements prévu et observé doivent faire l'objet d'une analyse.

Notons au passage que le terme ( seuil », souvent utilisé dans la pratique de préférence à celui de « limites », préconisé par l'Eurocode 7, ne serait en toute rigueur approprié que dans un monde virtuel où le comportement géotechnique pourrait être décrit par la mesure d'un paramètre unique, et doit donc être considéré comme une simple commodité de langage.

Parmi les limites du « raisonnable » figure notamment celle, clairement explicitée dans l'Eurocode 7 cette fois-ci, d'un comportement fragile de l'ouvrage, ce dont la conception doit permettre d'éviter l'occurrence, ne serait-ce que parce qu'elle rend toute anticipation impossible et exclut donc le recours à cette méthode sécurisante qu'est le dimensionnement interactif.

L'exemple du métro de Rennes (Schlosser et Schmitt, 2007), au cours duquel le suivi géotechnique a mis en évidence des sollicitations plus importantes que prévu, montre qu'il n'a été possible d'intervenir, en ajoutant rapidement des butons supplémentaires, que parce que le dispositif d'appui comportait une lierne de répartition continue qui, même sur-sollicitée, permettait une redistribution d'efforts suffisante pour éviter, précisément, l'occurrence d'une rupture fragile.

Les états limites géotechniques associés à la stabilité générale, quant à eux, doivent être détectés non seulement par des analyses, mais encore si possible par des observations et des mesures du comportement antérieur du massif : un risque de sous-dimensionnement notable peut en effet résulter de la non prise en compte des sollicitations appliquées à un ouvrage amené à jouer, malgré lui, un rôle de stabilisation d'un glissement de terrain vis-à-vis duquel il n'aurait pas été dimensionné (Fig. 43).

Les facteurs partiels ont donc ici pour objet, non seulement de garantir un éloignement raisonnable visà-vis de l’ELU, mais encore de limiter les sollicitations appliquées aux ouvrages aux valeurs pour lesquelles ils ont été calculés : c'est la raison pour laquelle une saine pratique consiste à dissocier dans la mesure du possible les fonctions confortement et soutènement (Fig. 44), et à retenir, comme le font les normes françaises d'application de l'Eurocode 7 (AFNOR, 2009), des facteurs partiels plus élevés vis-à-vis de surfaces de rupture proche d'ouvrages sensibles aux déformations.

On concevrait mal, là encore, que des projets soumis à un risque potentiel d'instabilité générale ne fassent pas l'objet, au minimum d'un suivi géotechnique dans la mesure où le respect des facteurs règlementaires permet de les classer en catégorie 2 au sens de l'Eurocode 7, voire d'un dimensionnement interactif obligatoire dans le cas d'un ouvrage de catégorie 3.

Enfin les états limites hydrauliques, souvent assimilables à des ruptures fragiles (ceux qui les ont vécus n'ignorent pas la rapidité avec laquelle surviennent et se développent les phénomènes de ( renard )), peuvent néanmoins être détectés au moyen d'observations visuelles des arrivées d'eau en fond de fouille, et bien évidemment d'un suivi attentif des débits de pompage
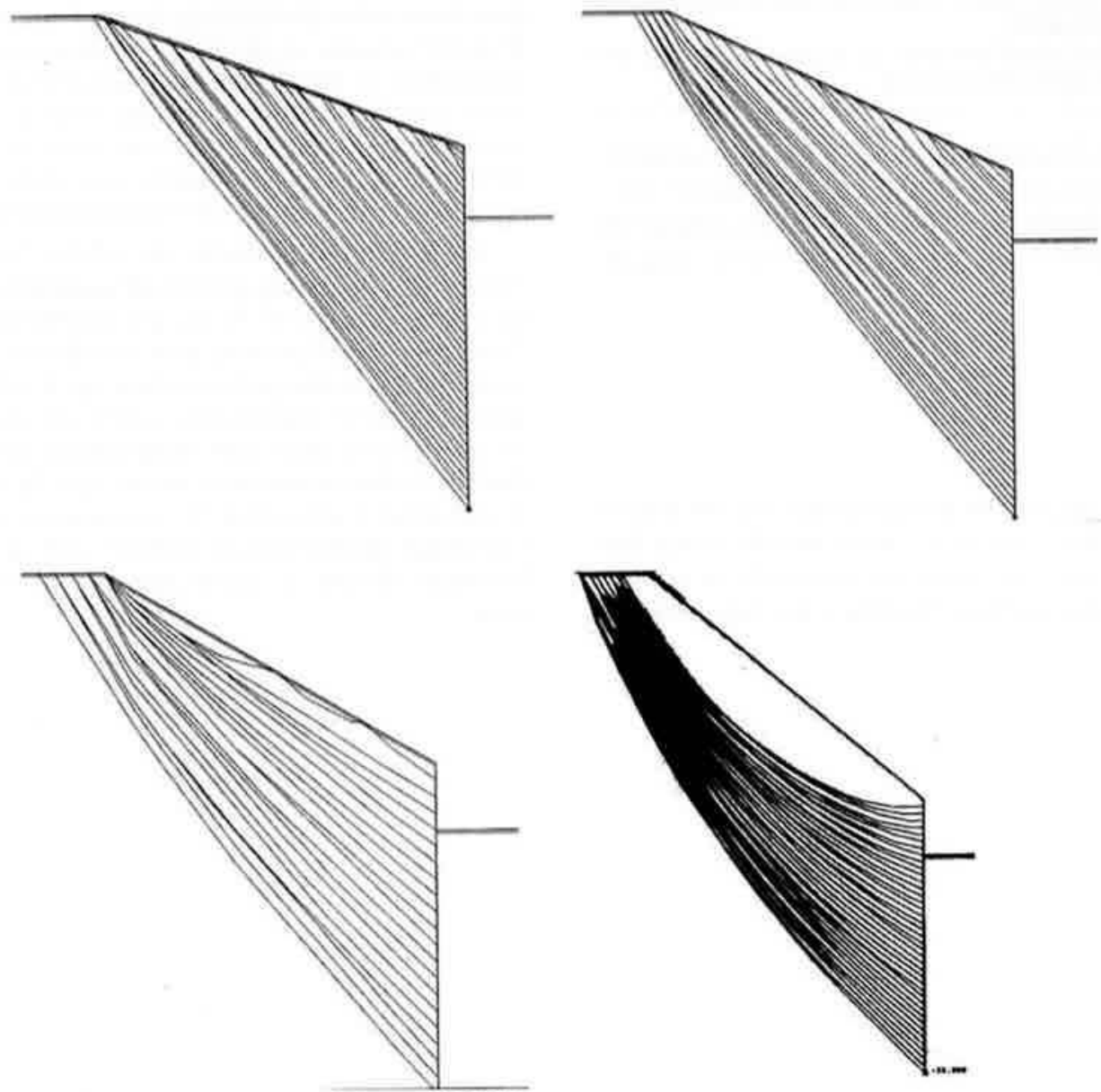

FG. 43 Du soutènement des terres à la stabilité des pentes.

From earth retaining to slope stability problems. 


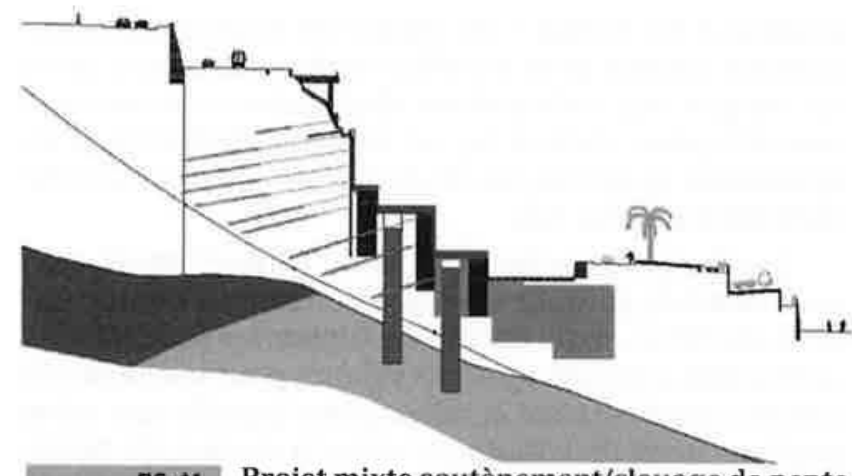

56.44 Projet mixte soutènement/clouage de pente à Monaco.

Mixed retaining structure/slope nailing project in Monaco.

\begin{tabular}{|c|c|c|}
\hline TYPE D'INSÉCURITÉ & ORIGINE & SUIVI \\
\hline \multicolumn{3}{|l|}{ États limites de service } \\
\hline & $\begin{array}{l}\text { - Réalisation de l'écran } \\
\text { - Déplacement de l'écran }\end{array}$ & $\begin{array}{l}\text { Topographie } \\
\text { Inclinométrie }\end{array}$ \\
\hline Déformations & - Rabattement de la nappe & Piézométrie \\
\hline \multicolumn{3}{|l|}{ États limites ultimes } \\
\hline - Défaut de butée & $\begin{array}{l}\text { - Hétérogénéité } \\
\text { - Dégradation } \\
\text { - Gradient hydraulioue }\end{array}$ & $\begin{array}{l}\text { Inclinométrie } \\
\text { Piézométrie }\end{array}$ \\
\hline - Défaut de portance & & $\begin{array}{l}\text { Topographie } \\
\text { Inclinométrie }\end{array}$ \\
\hline - Instabilité d'ensemble & $\begin{array}{l}\text { - Instabilité initiale du site } \\
\text { - Effet des travaux }\end{array}$ & $\begin{array}{l}\text { Extensométrie } \\
\text { Observations } \\
\text { Piézométrie }\end{array}$ \\
\hline - Instabilité hydraulique & - Piézométrie & Débits \\
\hline - Instabilité structurale & $\begin{array}{l}\text { - Action géotechnique } \\
\text { - Dispositions constructives }\end{array}$ & Inclinométrie \\
\hline
\end{tabular}

Fic. 45 Relation entre état limite potentiel et suivi préventif.

Relationship between anticipated limit state and preventive monitoring.

et relevés piézométriques au cours des terrassements, lesquels s'accompagnent d'une augmentation progressive et pas nécessairement linéaire des gradients hydrauliques, parfois à l'origine de sinistres importants.

\section{5}

\section{Conclusion}

Les exemples présentés avaient pour but de mettre en évidence l'intérêt du suivi, sous ses diverses formes, interactives ou non, pour permettre à ces « aventuriers ») que sont, parfois malgré eux, les concep-

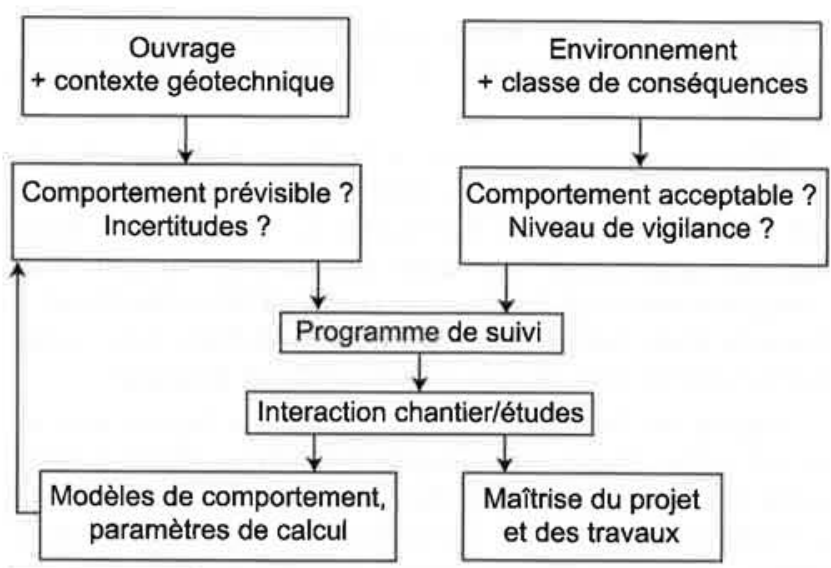

Fl. 46 Application delaméthodeobservationnelle logigramme.

Application of the observational method process flow diagram.

teurs d'ouvrages géotechniques, de maîtriser les deux aspects fondamentaux du dimensionnement que sont les déplacements et la sécurité, ce que les calculs géotechniques ne permettent pas toujours, eu égard non seulement à la complexité croissante des projets mais encore, et surtout, à l'éternelle complexité de ce matériau toujours surprenant qu'est le sol.

Les logigrammes des figures 45 et 46 résument une démarche ( observationnelle » adaptée à la maîtrise des risques et incertitudes inhérentes à l'exercice de la géotechnique.

On peut imaginer que le recours au suivi géotechnique deviendra caduque le jour où un nombre suffisant d'observations et de rétro-analyses aura permis aux praticiens de répondre avec assurance aux deux questions fondamentales qui sont celle du comportement prévisible de l'ouvrage et celle, encore plus redoutable, de la précision avec laquelle on estime être réellement en mesure de prévoir ce comportement.

La précision actuelle du calcul des déplacements étant d'un ordre de grandeur assurément plus proche de $100 \%$ que de $10 \%$, ce qui explique que l'Eurocode 7 indique explicitement que ce calcul « ne doit pas être considéré comme précis ») mais qu'il « donne seulement une indication approchée »), il n'est pas déraisonnable de penser que quelques générations de géotechniciens devront encore œuvrer avant que le suivi géotechnique puisse disparaître de la pratique courante, et que l'ouvrage géotechnique puisse être dimensionné avec le même degré de certitude que le reste de la structure. 
AFNOR - Norme française NF P 94-282, Calcul géotechnique. Ouvrages de soutènement. Ecrans, 2009

AFNOR - Norme française NF P 94-500, Missions d'ingénierie géotechnique. Classification et spécifications, 2006.

Allagnat D. - La méthode observationnelle pour le dimensionnement interactif des ouvrages. Presses des Ponts et Chaussées, 2005.

Azoune L., 2002 - Comparaison entre les modèles de comportement des sols sur Plaxis et application pour les ouvrages de Trémie Pasteur et Colombes. Rapport de stage de DEA, Ecole centrale Paris, 2002.

Bazin E., Schmitt P. - Analyse des mesures de déformation des parois moulées du métro du Caire. $X V^{*} C I M S G$, Istanbul, vol. 2, 2001.

Bustamante M., Gouvenot D. - Mesures in situ sur les ouvrages maritimes de soutènement. Annales de I'TTBTP, $n^{\circ} 375$ Série Sols et Fondations, $n^{\circ} 167,1978$.

Bourdon C., Ménard L. - Calcul des rideaux de soutènement. Méthode nouvelle prenant en compte les conditions réelles d'encastrement. Sols-Soils, $n^{\circ} 12,1965$.

Cambefort H. - Injection des sols. Principes et méthodes. Eyrolles, 1967.

Combarieu O. - L'usage des modules de déformation en géotechnique. Revue française de géotechnique, $\mathrm{n}^{\circ}$ 114, 2006.

Delattre L., Joignant P., Lavisse J., Marten S., Pioline M., Vinceslas G. - Comportement observé du mur de quai du nouveau Port 2000, Le Havre. XVI CIMSG, Osaka, vol. 3, 2005.

Ducas V. - Comportement expérimental des parois moulées sur le chantier Trémie Pasteur à Rouen. Thèse de doctorat, Laboratoire central des ponts et chaussées, 2001.

Escobar M.P.G, - Utilisation du programme Plaxis pour les ouvrages de soutènement : Interprétation des mesures des parois moulées sur le chantier de la Trémie Pasteur à Rouen. Rapport de stage de DEA, Ecole centrale Paris, 2001.

Eurocode 7 - NF EN 1997, Calcul géotechnique. AFNOR, 2005.

Gambin M. - Reconnaissance des terrains in situ : essai pressiométrique. Traité MIM, Série Géomatériaux, 2005.

Holtz R.D., Magnan J.-P., Schlosser F. Construction géotechnique. XIe CIMSG, vol. 1, San Francisco, 1985.

Lavisse J., Moreau T., Robert J., Schmitt P. Le dimensionnement interactif pour la réalisation d'une fouille profonde sur le flanc d'un versant fortement urbanisé à Monaco. XIV CEMSG, Madrid, 2007.

Lavisse J., Schmitt P. - Interprétation de mesures de tassement sous des remblais de préchargement au Port de Rouen. Congrès ASEP-GI, Paris, 2004.

Londez M., Namur S., Schmitt P. - Analyse des mesures de déformations d'une paroi moulée à Colombes. XIV CIMSG, Hambourg, vol. 2, 1997

Marten S. - Etude expérimentale et méthodologique sur le comportement des écrans de soutènement. Thèse de doctorat, École nationale des ponts et chaussées, 2005.

Ménard L. - Règles d'utilisation des techniques pressiométriques et d'exploitation des résultats obtenus pour le calcul des fondations. Notice générale D60, 1965.

Nguyen P.D. - Modélisation numérique des soutènements d'excavations. Thèse de doctorat, École nationale des ponts et chaussées, 2003

Phienwej N., Hock G.C., Balasubramnaiam A.B. - Ground movements in deep excavations with concrete diaphragm walls in Bangkok soils. XII't South East Asian Geotechnical Conference, Taipei, Taiwan, 1998.

Plumelle C., Serrai K., Schmitt P. - Interprétation par la méthode des éléments finis des mesures de déformations d'une paroi moulée à Colombes. XVI CIMSG, Osaka, vol. 2, 2005

Reynaud P.-Y., Rivière P. - Mesure des pressions développées dans une parol moulée en cours de bétonnage. Bulletin de liaison des laboratoires des ponts et chaussées, $\mathrm{n}^{\circ} 113,1981$

Sayavong S. - Mur de quai du Havre « Port 2000 » en paroi moulée : analyse et recalage du comportement donné par le calcul par rapport au comportement réellement observé. Mémoire de TFE, École spéciale des travaux publics, 2008

Schlosser F - Le renforcement des sols et le multi-ancrage pour les grandes excavations. Forum praticiens/universitaires XIV CEMSG, Madrid, 2007.

Schlosser F., Schmitt, P. - La méthode observationnelle: du suivi géotechnique au dimensionnement interactif. Travaux, $n^{\circ} 844,2007$.

Schmitt P. - Etude expérimentale de la sollicitation exercée par le sol sur les ouvrages de soutènement souple. Revue française de géotechnique, $\mathrm{n}^{\circ} 28,1984$

Schmitt P. - Méthode empirique d'évaluation du coefficient de réaction du sol vis-à-vis des ouvrages de soutènement souples. Revue française de géotechnique, $\mathrm{n}^{\circ} 71,1995$

Schmitt P. - De l'élasticité linéaire au coefficient de réaction : théories, observations et ordres de grandeur. Revue française de géotechnique, $\mathrm{n}^{\circ} 85,1998$

Serrai K. - Différentes méthodes de calculs des écrans plans. Comparaisons des résultats avec des mesures de chantier. Thèse de doctorat, Conservatoire national des arts et métiers, 2001.

Terzaghi K. - Evaluation of coefficients of subgrade reaction. Geotechnique, vol. 5 , 1955

Westergaard H.M - Stresses in concrete pavements computed by theoretical analysis. Public Roads, $n^{\circ} 7,1926$ 